\title{
Combined effects of NPK fertilizer with foliar application of benzyladenine or gibberellic acid on Dracaena marginata 'Bicolor' grown in different potting media
}

\author{
Hossam Ahmed Ashour ${ }^{1}$ (), Asmaa Bader Eldeen El- Attar ${ }^{1}\left(\mathbb{D}\right.$, Mohamed Mahmoud Abdel Wahab $^{2}$ (]) \\ ${ }^{1}$ Cairo University, Faculty of Agriculture, Ornamental Horticulture Department, Giza, Egypt. \\ ${ }^{2}$ Cairo University, Faculty of Agriculture, Plant Physiology Department, Giza, Egypt.
}

\begin{abstract}
A pot experiments was carried out to evaluate the influence of growing media and combined treatments of NPK with either benzyladenine or gibberellic acid on growth, chemical constituents and anatomical structure of Dracaena marginata 'Bicolor'. The plants were grown in two growing media; peat-moss, peat-moss + sand $\left(1: 1, \mathrm{v} \mathrm{v}^{-1}\right)$, received monthly NPK fertilizers $\left(2\right.$ and $\left.4 \mathrm{~g} \mathrm{pot}^{-1}\right)$ combined with either of benzyl adenine (BA) at 100 and $150 \mathrm{ppm}$ or gibberellic acid $\left(\mathrm{GA}_{3}\right)$ at 150 and $250 \mathrm{ppm}$, while the control plants received no treatments. As general, the results indicated that, peat- moss was superior to peat-moss + sand medium on increasing most of vegetative growth parameters in terms of plant height, number of leaves/plant, leaf area, root length, as well as fresh and dry weights of leaves, stems and roots/plant, besides some macro elements represented in $\mathrm{N}, \mathrm{P}, \mathrm{K}, \mathrm{Ca}$ and $\mathrm{Mg} \%$ in both leaves and stems. While plants grown in peat-moss + sand possessed significantly higher contents of total chlorophylls, total carbohydrates, $\mathrm{Cu}, \mathrm{Fe}, \mathrm{Mn}, \mathrm{Zn}$ and $\mathrm{B}$ than those grown in peat- moss alone. Plants received combined NPK with either BA or GA 3 resulted in significant increases in most of morphological and chemicals content over the control plants and it was outstanding that, $\mathrm{GA}_{3}$ was more effective than BA when they were combined with NPK. It can be concluded that for the highest quality, quantity growth and economic production of Dracaena marginata 'Bicolor', the plants could be grown in a medium of peat-moss and supplied monthly with NPK fertilizer at $2 \mathrm{~g}$ plant ${ }^{-1}$ along with foliar sprayed with $250 \mathrm{ppm}^{\mathrm{GA}} \mathrm{A}_{3}$
\end{abstract}

Keywords: foliage plant, hormones, mineral nutrition, peat moss, sand.

\section{Resumo}

Efeito combinado do fertilizante NPK com a aplicação foliar de benziladenina ou ácido giberélico em Dracaena marginata' Bicolor 'cultivada em diferentes substratos

Um experimento em vaso foi realizado para avaliar a influência do substrato e dos tratamentos combinados de NPK com benziladenina ou ácido giberélico no crescimento, constituintes químicos e estrutura anatômica de Dracaena marginata 'Bicolor'. As plantas foram cultivadas em dois meios de cultivo; turfa-musgo, turfa-musgo + areia $\left(1: 1, \mathrm{v} \mathrm{v}^{-1}\right)$, recebeu fertilizantes NPK mensais (2 e $4 \mathrm{~g} \mathrm{vaso}^{-1}$ ) combinados com benziladenina (BA) a 100 e $150 \mathrm{ppm}$ ou ácido giberélico $\left(\mathrm{GA}_{3}\right.$ ) a 150 e 250 ppm, enquanto as plantas controle não receberam tratamentos. De modo geral, os resultados indicaram que turfa-musgo foi superior a turfa-musgo + areia média no aumento da maioria dos parâmetros de crescimento vegetativo em termos de altura da planta, número de folhas/ planta, área foliar, comprimento da raiz, bem como fresco e seco pesos de folhas, caules e raízes/planta, além de alguns macroelementos representados em \% de N, P, K, Ca e Mg nas folhas e caules. Enquanto as plantas cultivadas em turfa + areia possuíam conteúdos significativamente maiores de clorofilas totais, carboidratos totais, $\mathrm{Cu}, \mathrm{Fe}, \mathrm{Mn}, \mathrm{Zn}$ e B do que aquelas cultivadas apenas em turfa. As plantas que receberam NPK combinado com BA ou GA 3 resultaram em aumentos significativos na maioria do conteúdo morfológico e químico sobre as plantas de controle e foi notável que $\mathrm{GA}_{3}$ foi mais eficaz do que BA quando combinados com NPK. Pode-se concluir que para a mais alta qualidade, crescimento em quantidade e produção econômica de Dracaena marginata 'Bicolor', as plantas poderiam ser cultivadas em meio de turfa e abastecidas mensalmente com fertilizante NPK a $2 \mathrm{~g}$ planta ${ }^{-1}$ junto com pulverização foliar de $\mathrm{GA}_{3}$ a $250 \mathrm{ppm}$.

Palavras-chave: folhagem, hormônios, nutrição mineral, turfa, areia.

\footnotetext{
* Corresponding author: hossam.ahmed@agr.cu.edu.eg
} 


\section{Introduction}

The genus Dracaena comprising about 40 species belongs to the family Ruscaceae, only six species are cultivated and used as foliage plants including $D$. deremensis, D. fragrans, D. reflex, D. sanderiana, D. marginata and D. surculosa. Dracaena marginata known as Madagascar dragon is evergreen ornamental shrub or small tree. It is growing to 3-5 $\mathrm{m}$ tall with a spread of $0.9-1.2$ $\mathrm{m}$. The leaves are slender, sword-shaped, slightly flexible having pleasant appearance (Huxley, 1992; Odenwald and Turner, 2006).

Growing media plays a vital role in plant growth and development of indoor potted plants. The physical and chemical properties of used media are the dominant factors affecting plant growth; hence these properties influence availability of nutrients and water for the plant growth and penetration of its roots in the soil. Peat moss is the most widely organic substrate used for the preparation of growing media for the potted ornamental plants. However, it is very expensive so that its replacement with cheaper available substrates is necessary (Shahid et al., 2017). Sand is generally the least expensive of inorganic amendments added to growing media for achieving and maintaining a structural system of macro pores that improve aeration and drainage. It was found that peatmoss alone increased the growth of different indoor plants (Mousa et al., 2015; Younis et al., 2016; Badran et al., 2017), while other studies demonstrated that peat + sand $(2: 1)$ medium increased the growth and chemical composition parameters of Celosia argentea (Abd el Gayed and Attia, 2018).

Fertilizers are essential for production of healthy potted ornamental plants. The NPK fertilization has a positive effect on the various biochemical processes that occur within the plant giving normal plant growth and development. Different NPK fertilization treatments have favorably influenced on growth of several foliage plants (El-Naggar and Ahmad, 2016; Abou Dahab et al., 2017; ElSayed and Ismail, 2017; Mohamed, 2018).

Concerning Cytokinins are a large group of plant hormones which play a pivotal role as active molecules in many physiological processes of plant growth and development. Benzyl adenine (BA) had been newly used to maintain or increase the quality of different ornamental plants (Askari-Khorasgani and Mortazaeinezhad 2016; Matak et al., 2017), Moreover, its ability to increase number of chloroplasts, chlorophyll synthesis, bud differentiation and branching had been reported (Kochhar and Sukhbir, 2020). It was reported that its application induced vegetative growth, photosynthesis rate, soluble sugars, indoles, soluble phenols and the contents of $\mathrm{N}, \mathrm{P}$ and $\mathrm{K}$ in organs of croton plants (Ibrahim et al., 2010). The leaf area and fresh weight of leaves and stems had been recorded (Fuadi et al., 2014).

Gibberellins (GAs) can influence different biological processes and plant growth, i.e. from vegetative growth to flowering, and also stimulate aspects of flower development. In addition, gibberellins effect on other developmental responses such as dormancy, sex expression, leaf and fruit senescence, root and fruit growth and development of seeds in the fruit (Plackett and Wilson, 2016; Satish and Manju, 2018). The beneficial effect of $\mathrm{GA}_{3}$ on different indoor plants had been reported by prior research who concluded that $\mathrm{GA}_{3}$ induced flowering (Huang et al., 2015; Gad et al., 2016) promoted plant height, leaf area, number of leaves, stem diameter, fresh and dry weights, relative growth rate as well as increased the contents of total chlorophylls, total carbohydrates, and macronutrients (Mazher et al., 2014; Hananfy et al., 2019).

Furthermore, previous studies had been conducted in order to evaluate the interactive effect of BA with $\mathrm{GA}_{3}$ (Henschke et al., 2015; Gabrel et al., 2018; Sardoei, 2018), NPK fertilizer with BA (Ngapui et al., 2018), NPK with $\mathrm{GA}_{3}$ (El-Sayed et al., 2016) and NPK with potting media (Badran et al., 2017; Mohamed, 2018). However, there is lake of researches about the influence of potting media and NPK along with BA or $\mathrm{GA}_{3}$ as growth regulators on the indoor plants and their anatomical structure.

Therefore, the main object of present study was to determine the effect of different growing media and combined NPK with either BA or $\mathrm{GA}_{3}$ and their interaction effects on the growth, chemical constituents and anatomical structure of Dracaena marginata 'bicolor' plants.

\section{Material and Methods}

The experiment was carried out in the glasshouse at the nursery of Ornamental Horticulture Department, Faculty of Agriculture, Cairo University, Giza, Egypt during the two successive seasons of 2018 and 2019.

Seedlings of Dracaena marginata 'Bicolor' plants were obtained from the Ornamental Department, Agriculture Research Centre A.R.C, Ministry of Agriculture, Giza, Egypt. Uniformal seedlings (20-25 height $\mathrm{cm}$ and having 10-15 leaves/ seedlings) were planted individually On $20^{\text {th }}$ March 2018 and 2019, in $30 \mathrm{~cm}$ plastic pots filled media containing peat-moss or peat-moss + sand $\left(1: 1: \mathrm{v} \mathrm{v}^{-1}\right)$ media. The chemical analysis of growing media was done at Soil, Water and Environment Research Institute, Agriculture Research Centre A.R.C according to (Jackson 1973), the results are presented in Table 1 . 
Table 1. The properties and constituents of growing media used for growing Dracaena marginata during 2018 and 2019 seasons.

\begin{tabular}{|c|c|c|c|c|c|c|c|c|c|c|c|c|c|}
\hline \multirow{2}{*}{ Growing media } & \multirow{2}{*}{$\begin{array}{c}\text { Organic } \\
\text { matter } \\
{[\%]}\end{array}$} & \multirow{2}{*}{$\begin{array}{c}\text { EC } \\
{[d S / m]}\end{array}$} & \multirow{2}{*}{$\mathbf{p H}$} & \multirow{2}{*}{$\begin{array}{c}\text { W H C } \\
{[\%]}\end{array}$} & \multicolumn{5}{|c|}{ Macro nutrients [\%] } & \multicolumn{4}{|c|}{ Micro nutrients [ppm] } \\
\hline & & & & & $\mathbf{N}$ & $\mathbf{P}$ & $\mathbf{K}$ & $\mathrm{Mg}$ & $\mathrm{Ca}$ & $\mathrm{Fe}$ & Mn & Zn & $\mathrm{Cu}$ \\
\hline Peat moss & 92 & 0.57 & 5.74 & 61.5 & 1.34 & 0.24 & 0.76 & 0.23 & 1.02 & 427 & 127 & 45.44 & 8.73 \\
\hline Peat + sand $(1: 1)$ & 42.63 & 0.97 & 6.97 & 45.7 & 1.11 & 0.11 & 0.53 & 0.41 & 1.83 & 456 & 114 & 40.04 & 5.66 \\
\hline
\end{tabular}

WHC: Water holding capacity

Starting from $20^{\text {th }}$ April till $20^{\text {th }}$ October, for both seasons, the plants grown in different soil media received monthly different treatments. A combination of NPK was added to soil in forms of (NPK, 20:20:20) at the rate of 2 and 4 g pot $^{-1}$ with foliar spray of either benzyladenine (BA) at the concentrations of $(100,150 \mathrm{ppm})$ or gibberellic acid $\left(\mathrm{GA}_{3}\right)$ at the concentrations of $(150,250 \mathrm{ppm})$ until dropping point. The control plants were sprayed only with tap water without fertilization.

The experimental design was factorial $2 \times 9$ in randomized complete block design with 18 treatments. The first factor is 2 growing media, peat-moss and peatmoss + sand $(1: 1 \mathrm{v} / \mathrm{v})$. The second factor is 9 treatments of combined NPK with either BA or $\mathrm{GA}_{3}$ concentrations including control (T1), $2 \mathrm{~g} \mathrm{NPK}+\mathrm{BA}$ at $100 \mathrm{ppm}$ (T2), 2 $\mathrm{g} \mathrm{NPK}+\mathrm{BA}$ at $150 \mathrm{ppm}$ (T3), $4 \mathrm{~g} \mathrm{NPK}+\mathrm{BA}$ at $100 \mathrm{ppm}$ (T4), $4 \mathrm{~g} \mathrm{NPK}+\mathrm{BA}$ at $150 \mathrm{ppm}$ (T5), $2 \mathrm{~g} \mathrm{NPK}+\mathrm{GA}_{3}$ at $150 \mathrm{ppm}$ (T6), $2 \mathrm{~g} \mathrm{NPK}+\mathrm{GA}_{3}$ at $250 \mathrm{ppm}$ (T7), $4 \mathrm{~g}$ $\mathrm{NPK}+\mathrm{GA}_{3} 150 \mathrm{ppm}$ (T8), $4 \mathrm{NPK}+\mathrm{GA}_{3}$ at $250 \mathrm{ppm}$ (T9). Each treatment consisting of 6 pots arranged in 3 blocks (replicates), each replicate including 36 plants (2 plants from each treatment).

On $20^{\text {th }}$ November for both seasons, the experiment was terminated and the vegetative growth traits were registered, including: plant height $(\mathrm{cm})$, number of leaves/plant, stem diameter $(\mathrm{mm})$, leaf area $\left(\mathrm{cm}^{2}\right)$, root length $(\mathrm{cm})$, fresh and dry weights of leaves, stems and roots/plant. The chemical constituents were determined including: total chlorophylls (SPAD) in fresh leaf samples (Netto et al., 2005), total carbohydrates (\%) in dried leaves and stems samples (Dubois et al., 1956). Another dried leaves and stems samples were digested for nutrients determination. The concentration of $\mathrm{N}$ (Horneck and Miller, 1998), $\mathrm{P}$ (Jackson, 1973), K (Horneck and Hanson, 1998), Ca and $\mathrm{Mg}$ (Estefan et al., 2013) and the contents of $\mathrm{Cu}, \mathrm{Fe}, \mathrm{Mn}$ and Zn (Chapman and Pratt 1961), beside B (Gupta, 1998) were determined.

\section{Anatomical studies}

At the end of vegetative growth, specimens of the leaves were taken and tissue specimens dissected to the size of the sample, and transferred to FAA solution at room temperature for 20 hours. After fixation, they were washed in $50 \%$ ethanol then dehydration was completed by transferring the sample to $100 \%$ butyl alcohol (changes, for one day each). Dehydrated sample were immersed into a 1:1 solution of $100 \%$ ethanol: xylene for 1 hour, then transferred into xylene for storage until infiltration with molten Paraplast in a $55-60^{\circ} \mathrm{C}$ oven. The tissues were placed into wax $58^{\circ} \mathrm{C}$ and placed into embedding molds in 7x7 $55 \mathrm{~mm}$ disposable base molds (SLEE MAINS type MPS/P, Germany). The wax blocks containing the embedded tissue were sectioned using a rotary microtome (Leica RM 2125) with heavy duty high profile disposable microtome blades. (Ellis, 1976; Aiken et al., 1984). Images were acquired with a camera Leica ICC50 HD digital camera attached to a Leica motorized light microscope system. Averages of readings from 4 slides / treatment were calculated.

All previous data were subjected to analysis of variance (ANOVA). The effects of growing media $(\mathrm{G})$, combined treatments of NPK with either BA or $\mathrm{GA}_{3}$ concentrations (T), and their interactions was analyzed by two-ways randomized block ANOVA. The Least Significant Difference (L.S.D.) test at the 5\% level was used to compare the means values of different parameters (Steel and Torrie, 1997).

\section{Results and Discussion}

\section{Vegetative growth parameters}

\section{Effect of growing media}

The results of the two growing seasons (Tables 2- 4) showed that, in most cases, the plants grown in peat-moss had significantly higher values for most vegetative growth parameters of Dracaena marginata 'Bicolor' as compared with that grown in peat moss + sand. The only exception to this general trend was recorded with stem diameter which recorded higher increment with plants grown in peat moss + sand than those grown in peat moss alone. The results of superior peat moss alone as a growing medium in boosting the vegetative traits are in harmony with those reported on indoor plants by previous studies (Mousa et al., 2015; Younis et al., 2016; Badran et al., 2017). 
Table 2. Effect of growing media, combined NPK with foliar application of plant growth regulators treatments and the interactions between the two factors on plant height, number of leaves, stem diameter, leaf area and root length of Dracaena marginata during the 2018 and 2019 seasons.

\begin{tabular}{|c|c|c|c|c|c|c|c|c|c|c|c|c|c|c|c|}
\hline \multirow{4}{*}{$\begin{array}{l}\text { Treatments } \\
\text { (T) }{ }^{1}\end{array}$} & \multicolumn{15}{|c|}{$1^{\text {st }}$ season } \\
\hline & \multicolumn{15}{|c|}{ Growing media (G) } \\
\hline & Peat & Peat + sand & Mean $(T)$ & Peat & Peat+ sand & Mean $(T)$ & Peat & Peat+ sand & Mean (T) & Peat & Peat + sand & $\operatorname{Mean}(\mathrm{T})$ & Peat & Peat+ sand & Mean $(\mathrm{T})$ \\
\hline & \multicolumn{3}{|c|}{ Plant height $[\mathrm{cm}]$} & \multicolumn{3}{|c|}{ Number of leaves /plant } & \multicolumn{3}{|c|}{ Stem diameter [mm] } & \multicolumn{3}{|c|}{ Leaf area $\left[\mathrm{cm}^{2}\right]$} & \multicolumn{3}{|c|}{ Root length [cm] } \\
\hline T1 (Control) & $43.39 \mathrm{e}-\mathrm{gA}$ & $39.41 \mathrm{gA}$ & 41.40 & $39.33 \mathrm{iA}$ & $39.10 \mathrm{iA}$ & 39.22 & 1.08 & 1.21 & $1.15 \mathrm{a}$ & $53.53 \mathrm{gA}$ & $50.67 \mathrm{gA}$ & 52.10 & $52.13 \mathrm{hA}$ & $43.86 \mathrm{iB}$ & 48.00 \\
\hline $\mathrm{T} 2$ & $50.87 \mathrm{~b}-\mathrm{dA}$ & $43.49 \mathrm{e}-\mathrm{gB}$ & 47.18 & $48.80 \mathrm{~d}-\mathrm{gA}$ & $49.20 \mathrm{~d}-\mathrm{fA}$ & 49.00 & 1.16 & 1.21 & $1.19 \mathrm{a}$ & $75.45 \mathrm{efA}$ & $68.42 \mathrm{fA}$ & 71.94 & $60.70 \mathrm{~d}-\mathrm{fA}$ & $57.13 \mathrm{fgA}$ & 58.92 \\
\hline $\mathrm{T} 3$ & $54.71 \mathrm{abA}$ & $51.25 \mathrm{~b}-\mathrm{dA}$ & 52.98 & $51.13 \mathrm{cdA}$ & $53.06 \mathrm{bcA}$ & 52.10 & 1.22 & 1.27 & $1.25 \mathrm{a}$ & $82.45 \mathrm{deA}$ & $74.23 \mathrm{fB}$ & 78.34 & 59.70 efA & $61.46 \mathrm{~d}-\mathrm{fA}$ & 60.58 \\
\hline $\mathrm{T} 4$ & $49.62 \mathrm{~d}-\mathrm{gA}$ & $42.49 \mathrm{fgA}$ & 46.06 & $45.67 \mathrm{hA}$ & $46.06 \mathrm{ghA}$ & 45.87 & 1.21 & 1.30 & $1.26 \mathrm{a}$ & $70.47 \mathrm{fA}$ & $71.53 \mathrm{fA}$ & 71.00 & $69.63 \mathrm{abA}$ & $65.43 \mathrm{~b}-\mathrm{dA}$ & 67.53 \\
\hline T5 & $50.01 \mathrm{~b}-\mathrm{eA}$ & $47.09 \mathrm{c}-\mathrm{fA}$ & 48.55 & $47.17 \mathrm{f}-\mathrm{hA}$ & $49.73 \mathrm{~d}$-fA & 48.45 & 1.28 & 1.44 & $1.36 \mathrm{a}$ & $73.82 \mathrm{fA}$ & $75.33 \mathrm{fA}$ & 74.58 & $66.33 \mathrm{bcA}$ & 58.60 efB & 62.47 \\
\hline T6 & $53.89 \mathrm{bcA}$ & $51.28 \mathrm{~b}-\mathrm{dA}$ & 52.59 & $48.43 \mathrm{~d}-\mathrm{hA}$ & $49.93 \mathrm{~d}-\mathrm{fA}$ & 49.18 & 1.32 & 1.43 & $1.38 \mathrm{a}$ & $93.61 \mathrm{bcA}$ & $87.20 \mathrm{cdA}$ & 90.41 & $63.20 \mathrm{c}-\mathrm{eA}$ & $61.93 \mathrm{c}-\mathrm{fA}$ & 62.57 \\
\hline $\mathrm{T} 7$ & $60.62 \mathrm{aA}$ & $50.78 \mathrm{~b}-\mathrm{dB}$ & 55.70 & $57.10 \mathrm{aA}$ & $53.97 \mathrm{bB}$ & 55.54 & 1.30 & 1.50 & $1.40 \mathrm{a}$ & $106.01 \mathrm{aA}$ & $95.87 \mathrm{bB}$ & 100.94 & $72.16 \mathrm{aA}$ & $69.36 \mathrm{abA}$ & 70.76 \\
\hline $\mathrm{T} 8$ & $48.83 \mathrm{~b}-\mathrm{fA}$ & $51.60 \mathrm{~b}-\mathrm{dA}$ & 50.22 & $53.40 \mathrm{bcA}$ & $43.60 \mathrm{e}-\mathrm{hB}$ & 48.50 & 1.46 & 1.54 & $1.50 \mathrm{a}$ & $90.31 \mathrm{bcA}$ & $90.23 \mathrm{bcA}$ & 90.27 & $68.53 \mathrm{abA}$ & $53.86 \mathrm{ghB}$ & 61.20 \\
\hline T9 & 48.56 b-fA & 48.95 b-fA & 48.76 & $50.30 \mathrm{de} A$ & 49.20 d-fA & 49.75 & 1.32 & 1.51 & $1.42 \mathrm{a}$ & $94.14 \mathrm{bcA}$ & $94.64 \mathrm{bcA}$ & 94.39 & $69.43 \mathrm{abA}$ & $52.46 \mathrm{hB}$ & 60.95 \\
\hline Mean $(G)$ & 51.17 & 47.37 & ---- & 49.04 & 48.21 & ---- & $1.26 \mathrm{~B}$ & $1.38 \mathrm{~A}$ & ---- & 82.20 & 78.68 & ---- & 64.65 & 58.23 & ---- \\
\hline \multicolumn{16}{|l|}{ L.S.D. $(0.05)^{2}$} \\
\hline G & \multicolumn{3}{|c|}{ Sig. } & \multicolumn{3}{|c|}{ N.S } & \multicolumn{2}{|c|}{ Sig. } & \multicolumn{4}{|c|}{ Sig. } & \multicolumn{3}{|c|}{ Sig. } \\
\hline $\mathrm{T}$ & \multicolumn{3}{|c|}{4.31} & \multicolumn{3}{|c|}{1.84} & \multicolumn{2}{|c|}{1.62} & \multicolumn{4}{|c|}{5.02} & \multicolumn{3}{|c|}{3.03} \\
\hline GX T & \multicolumn{3}{|c|}{6.09} & \multicolumn{3}{|c|}{2.60} & \multicolumn{2}{|c|}{2.29} & \multicolumn{4}{|c|}{7.09} & \multicolumn{3}{|c|}{4.28} \\
\hline \multicolumn{16}{|c|}{$2^{\text {nd }}$ season } \\
\hline $\mathrm{T} 1$ (Control) & $44.99 \mathrm{jA}$ & $40.69 \mathrm{kB}$ & 42.84 & $41.20 \mathrm{iA}$ & $39.37 \mathrm{iA}$ & 40.29 & 1.11 & 1.19 & $1.15 \mathrm{a}$ & $58.59 \mathrm{iB}$ & $54.12 \mathrm{iB}$ & 56.36 & $49.80 \mathrm{jA}$ & $41.76 \mathrm{kB}$ & 45.78 \\
\hline $\mathrm{T} 2$ & $47.63 \mathrm{ijB}$ & $52.68 \mathrm{~d}-\mathrm{fA}$ & 50.16 & $51.86 \mathrm{~d}-\mathrm{fA}$ & $46.67 \mathrm{hB}$ & 49.27 & 1.09 & 1.16 & $1.13 \mathrm{a}$ & $81.75 \mathrm{ghA}$ & $75.24 \mathrm{hA}$ & 78.50 & $62.63 \mathrm{fgA}$ & $54.86 \mathrm{iB}$ & 58.75 \\
\hline $\mathrm{T} 3$ & $55.28 \mathrm{ceA}$ & $53.98 \mathrm{dfA}$ & 54.63 & 54.87 a-cA & $52.40 \mathrm{c}-\mathrm{fB}$ & 53.64 & 1.19 & 1.29 & $1.24 \mathrm{a}$ & $90.65 \mathrm{efA}$ & $85.81 \mathrm{fgA}$ & 88.23 & $65.66 \mathrm{deA}$ & $62.86 \mathrm{fgA}$ & 64.26 \\
\hline $\mathrm{T} 4$ & $48.68 \mathrm{hiA}$ & $46.65 \mathrm{ijA}$ & 47.67 & 51.33 efA & $45.37 \mathrm{fgB}$ & 48.35 & 1.24 & 1.52 & $1.38 \mathrm{a}$ & $82.05 \mathrm{ghA}$ & $77.14 \mathrm{hA}$ & 79.60 & $70.93 \mathrm{abA}$ & 63.83 e-gB & 67.38 \\
\hline T5 & $51.33 \mathrm{f}-\mathrm{hA}$ & $49.28 \mathrm{~g}-\mathrm{iA}$ & 50.31 & $54.03 \mathrm{~b}-\mathrm{dA}$ & $52.36 \mathrm{c}-\mathrm{fA}$ & 53.20 & 1.31 & 1.87 & $1.59 \mathrm{a}$ & $82.07 \mathrm{ghA}$ & $75.73 \mathrm{hA}$ & 78.90 & $68.93 \mathrm{bcA}$ & $55.30 \mathrm{iB}$ & 62.12 \\
\hline T6 & $58.04 \mathrm{a}-\mathrm{cA}$ & $56.02 \mathrm{~b}-\mathrm{dA}$ & 57.03 & $56.83 \mathrm{aB}$ & $54.13 \mathrm{~b}-\mathrm{dA}$ & 55.48 & 1.37 & 1.46 & $1.42 \mathrm{a}$ & $98.86 \mathrm{~b}-\mathrm{dA}$ & $97.10 \mathrm{c}-\mathrm{eA}$ & 97.98 & $66.60 \mathrm{cdA}$ & $58.66 \mathrm{hB}$ & 62.63 \\
\hline $\mathrm{T} 7$ & $60.54 \mathrm{aA}$ & $58.83 \mathrm{abA}$ & 59.69 & $56.83 \mathrm{aA}$ & $56.73 \mathrm{aA}$ & 56.78 & 1.45 & 1.34 & $1.40 \mathrm{a}$ & $107.08 \mathrm{aA}$ & 100.17a-cA & 103.63 & $72.60 \mathrm{a} A$ & $65.13 \mathrm{~d}-\mathrm{fB}$ & 68.87 \\
\hline $\mathrm{T} 8$ & $53.55 \mathrm{~d}-\mathrm{fA}$ & $52.61 \mathrm{~d}-\mathrm{fA}$ & 53.08 & $55.67 \mathrm{abA}$ & $52.43 \mathrm{c}-\mathrm{fB}$ & 54.05 & 1.42 & 1.89 & $1.66 \mathrm{a}$ & 91.67d-fA & 90.93d-fA & 91.30 & $69.33 \mathrm{bA}$ & $58.20 \mathrm{hB}$ & 63.77 \\
\hline T9 & $53.07 \mathrm{~d}-\mathrm{fA}$ & $52.37 \mathrm{e}-\mathrm{gA}$ & 52.72 & $53.56 \mathrm{~b}-\mathrm{eA}$ & $48.60 \mathrm{ghB}$ & 51.08 & 1.45 & 1.38 & $1.42 \mathrm{a}$ & $106.40 \mathrm{abA}$ & $97.22 \mathrm{c}-\mathrm{eB}$ & 101.81 & $70.90 \mathrm{abA}$ & $61.86 \mathrm{gB}$ & 66.38 \\
\hline Mean (G) & 52.57 & 51.46 & ---- & 52.91 & 49.78 & ---- & $1.29 \mathrm{~A}$ & $1.46 \mathrm{~B}$ & ---- & 88.79 & 83.72 & ---- & 66.38 & 58.05 & ---- \\
\hline L.S.D. $(0.05)^{*}$ & & & & & & & & & & & & & & & \\
\hline G & & N.S & & & Sig. & & & & & S & & & & Sig. & \\
\hline $\mathrm{T}$ & & 2.13 & & & 1.56 & & & & & 5 . & 15 & & & 1.66 & \\
\hline GX T & & 3.01 & & & 2.21 & & & 87 & & 7. & & & & 2.35 & \\
\hline
\end{tabular}

${ }^{1} \mathrm{~T} 1=$ Control, $\mathrm{T} 2=2 \mathrm{~g} \mathrm{NPK}+\mathrm{BA}(100 \mathrm{ppm}), \mathrm{T} 3=2 \mathrm{~g} \mathrm{NPK}+\mathrm{BA}(150 \mathrm{ppm}), \mathrm{T} 4=4 \mathrm{~g} \mathrm{NPK}+\mathrm{BA}(100 \mathrm{ppm}), \mathrm{T} 5=4 \mathrm{~g} \mathrm{NPK}+\mathrm{BA}(150 \mathrm{ppm}), \mathrm{T} 6=2 \mathrm{~g} \mathrm{NPK}+\mathrm{GA}{ }_{3}(150 \mathrm{ppm}), \mathrm{T} 7=2 \mathrm{~g} \mathrm{NPK}+\mathrm{GA}{ }_{3}(250 \mathrm{ppm}), \mathrm{T} 8=4 \mathrm{~g} \mathrm{NPK}+$ $\mathrm{GA}_{3}(150 \mathrm{ppm}), \mathrm{T} 9=4 \mathrm{~g} \mathrm{NPK}+\mathrm{GA}_{3}(250 \mathrm{ppm}),{ }^{2} \mathrm{G}=$ Growing media, $\mathrm{T}=$ Treatments, $\mathrm{G} \mathrm{X} \mathrm{T}=$ Growing media X Treatments, different lower case letters in the columns and upper case letters in the rows for each variable indicate a significant difference at $5 \%$ level of significance by LSD test. 


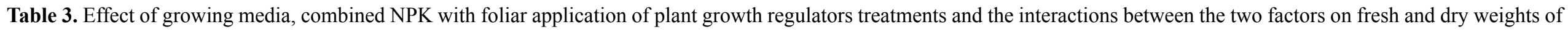
leaves and stems of Dracaena marginata during the 2018 and 2019 seasons.

\begin{tabular}{|c|c|c|c|c|c|c|c|c|c|c|c|c|}
\hline \multirow{3}{*}{ Treatments $(T)^{1}$} & \multicolumn{12}{|c|}{$1^{\text {st }}$ season } \\
\hline & \multicolumn{12}{|c|}{ Growing media (G) } \\
\hline & Peat & Peat+ sand & Mean $(\mathrm{T})$ & Peat & Peat+ sand & Mean $(\mathrm{T})$ & Peat & Peat + sand & Mean $(\mathrm{T})$ & Peat & Peat+ sand & Mean $(\mathrm{T})$ \\
\hline & \multicolumn{3}{|c|}{ F.W of leaves } & \multicolumn{3}{|c|}{ D.W of leaves } & \multicolumn{3}{|c|}{ F.W of stems } & \multicolumn{3}{|c|}{ D.W of stems } \\
\hline T1 (Control) & $41.94 \mathrm{fA}$ & $39.46 \mathrm{fA}$ & 40.70 & $9.42 \mathrm{eA}$ & $9.91 \mathrm{eA}$ & 9.67 & $34.75 \mathrm{ijA}$ & $31.88 \mathrm{jA}$ & 33.32 & $12.28 \mathrm{c}-\mathrm{eA}$ & $9.88 \mathrm{eB}$ & 11.08 \\
\hline $\mathrm{T} 2$ & $51.41 \mathrm{deA}$ & $51.39 \mathrm{deA}$ & 51.40 & $12.02 \mathrm{deA}$ & $14.33 \mathrm{a}-\mathrm{dA}$ & 13.18 & $37.67 \mathrm{f}-\mathrm{iA}$ & $37.35 \mathrm{~g}-\mathrm{iA}$ & 37.51 & $14.44 \mathrm{bcA}$ & $12.56 \mathrm{cdA}$ & 13.50 \\
\hline $\mathrm{T} 3$ & $56.82 \mathrm{c}-\mathrm{eA}$ & $52.79 \mathrm{c}-\mathrm{eA}$ & 54.81 & $15.69 \mathrm{a}-\mathrm{cA}$ & $14.98 \mathrm{a}-\mathrm{dA}$ & 15.34 & $50.43 \mathrm{abA}$ & $46.01 \mathrm{cdB}$ & 48.22 & $15.64 \mathrm{abA}$ & $13.49 \mathrm{~b}-\mathrm{dA}$ & 14.57 \\
\hline $\mathrm{T} 4$ & $52.56 \mathrm{c}-\mathrm{eA}$ & $50.14 \mathrm{eA}$ & 51.35 & 15.09 a-dA & $11.15 \mathrm{c}-\mathrm{eB}$ & 13.12 & $38.81 \mathrm{hiA}$ & $32.20 \mathrm{jB}$ & 35.51 & $12.76 \mathrm{cdA}$ & $12.63 \mathrm{cdA}$ & 12.70 \\
\hline $\mathrm{T} 5$ & $60.15 \mathrm{~b}-\mathrm{dA}$ & $50.14 \mathrm{eB}$ & 55.15 & $17.47 \mathrm{aA}$ & $13.15 \mathrm{abB}$ & 15.31 & 40.83 efA & $38.48 \mathrm{f}-\mathrm{hA}$ & 39.66 & $13.82 \mathrm{~b}-\mathrm{dA}$ & $12.63 \mathrm{cdA}$ & 13.23 \\
\hline T6 & $51.94 \mathrm{c}-\mathrm{eA}$ & $59.40 \mathrm{~b}-\mathrm{eA}$ & 55.67 & $12.62 \mathrm{c}-\mathrm{eB}$ & $16.37 \mathrm{~b}-\mathrm{dA}$ & 14.50 & $47.42 \mathrm{bcA}$ & $40.83 \mathrm{efB}$ & 44.13 & $14.58 \mathrm{bcA}$ & $13.80 \mathrm{~b}-\mathrm{dA}$ & 14.19 \\
\hline $\mathrm{T} 7$ & $72.08 \mathrm{aA}$ & $54.66 \mathrm{c}-\mathrm{eB}$ & 63.37 & $17.69 \mathrm{aA}$ & $15.97 \mathrm{a}-\mathrm{cA}$ & 16.83 & $52.50 \mathrm{aA}$ & $50.16 \mathrm{abA}$ & 51.33 & $17.38 \mathrm{aA}$ & $14.84 \mathrm{bcB}$ & 16.11 \\
\hline $\mathrm{T} 8$ & $56.38 \mathrm{abA}$ & $52.58 \mathrm{c}-\mathrm{eA}$ & 54.48 & $16.61 \mathrm{abA}$ & $10.32 \mathrm{c}-\mathrm{eB}$ & 13.47 & $40.22 \mathrm{e}-\mathrm{gA}$ & $38.46 \mathrm{f}-\mathrm{hA}$ & 39.34 & $13.81 \mathrm{~b}-\mathrm{dA}$ & $13.08 \mathrm{~b}-\mathrm{dA}$ & 13.45 \\
\hline T9 & $60.47 \mathrm{bcA}$ & $56.24 \mathrm{c}-\mathrm{eA}$ & 58.36 & $15.95 \mathrm{a}-\mathrm{cA}$ & $15.32 \mathrm{a}-\mathrm{dA}$ & 15.64 & $43.40 \mathrm{deA}$ & $36.09 \mathrm{hiB}$ & 39.75 & $13.38 \mathrm{~b}-\mathrm{dA}$ & $12.78 \mathrm{cdA}$ & 13.08 \\
\hline Mean (G) & 55.97 & 51.87 & --- & 14.73 & 13.50 & ---- & 42.89 & 39.05 & --- & 14.23 & 12.85 & --- \\
\hline \multicolumn{13}{|l|}{ L.S.D. $(0.05)^{2}$} \\
\hline G & \multicolumn{3}{|c|}{ N.S } & \multicolumn{3}{|c|}{ N.S } & \multicolumn{4}{|c|}{ Sig. } & \multicolumn{2}{|c|}{ Sig. } \\
\hline $\mathrm{T}$ & \multicolumn{3}{|c|}{5.35} & \multicolumn{3}{|c|}{2.13} & \multicolumn{4}{|c|}{2.10} & \multicolumn{2}{|c|}{1.57} \\
\hline $\mathrm{GXT}$ & \multicolumn{3}{|c|}{7.57} & \multicolumn{3}{|c|}{3.02} & \multicolumn{4}{|c|}{2.96} & \multicolumn{2}{|c|}{2.23} \\
\hline \multicolumn{13}{|c|}{$2^{\text {nd }}$ season } \\
\hline T1 (Control) & $43.82 \mathrm{hA}$ & $43.70 \mathrm{hA}$ & 43.76 & $10.91 \mathrm{gA}$ & $11.65 \mathrm{fgA}$ & 11.28 & $38.96 \mathrm{f}-\mathrm{hA}$ & $31.80 \mathrm{iB}$ & 35.38 & 13.84 a-dA & $11.12 \mathrm{dA}$ & 12.48 \\
\hline $\mathrm{T} 2$ & $51.20 \mathrm{gA}$ & $52.45 \mathrm{fgA}$ & 51.83 & $13.92 \mathrm{~d}-\mathrm{fA}$ & $14.86 \mathrm{c}-\mathrm{eA}$ & 14.39 & $35.45 \mathrm{hB}$ & $42.25 \mathrm{~d}-\mathrm{gA}$ & 38.85 & 14.31 a-dA & 15.51abA & 14.91 \\
\hline $\mathrm{T} 3$ & $58.33 \mathrm{~b}-\mathrm{fA}$ & $60.33 \mathrm{~b}-\mathrm{eA}$ & 59.33 & $16.67 \mathrm{~b}-\mathrm{dA}$ & $16.95 \mathrm{bcA}$ & 16.81 & $52.19 \mathrm{abA}$ & $46.71 \mathrm{cB}$ & 49.45 & $16.24 \mathrm{abA}$ & 13.79 a-dA & 15.02 \\
\hline $\mathrm{T} 4$ & $56.13 \mathrm{c}-\mathrm{gA}$ & $46.49 \mathrm{hB}$ & 51.31 & $16.39 \mathrm{~b}-\mathrm{eA}$ & $14.01 \mathrm{c}-\mathrm{eA}$ & 15.20 & $42.90 \mathrm{c}-\mathrm{fA}$ & $33.68 \mathrm{ghB}$ & 38.29 & $15.34 \mathrm{abA}$ & 13.97 a-dA & 14.66 \\
\hline T5 & 59.56b-fA & $52.05 \mathrm{~d}-\mathrm{gB}$ & 55.81 & $16.15 \mathrm{~b}-\mathrm{eA}$ & 17.14 bcA & 16.65 & $45.06 \mathrm{cdA}$ & $40.91 \mathrm{e}-\mathrm{gB}$ & 42.99 & $15.00 \mathrm{a}-\mathrm{cA}$ & 14.49 a-cA & 14.75 \\
\hline T6 & $54.40 \mathrm{e}-\mathrm{gA}$ & $60.47 \mathrm{~b}-\mathrm{eA}$ & 57.44 & $16.85 \mathrm{~b}-\mathrm{dA}$ & $14.73 \mathrm{c}-\mathrm{eA}$ & 15.79 & $46.42 \mathrm{cB}$ & $50.63 \mathrm{bA}$ & 48.53 & 14.99 a-cA & $14.91 \mathrm{a}-\mathrm{cA}$ & 14.95 \\
\hline $\mathrm{T} 7$ & $69.35 \mathrm{aA}$ & $65.39 \mathrm{abA}$ & 67.37 & $20.32 \mathrm{aA}$ & $18.38 \mathrm{abA}$ & 19.35 & $54.99 \mathrm{aA}$ & $44.78 \mathrm{c}-\mathrm{eB}$ & 49.89 & $17.60 \mathrm{aA}$ & $14.64 \mathrm{a}-\mathrm{cA}$ & 16.12 \\
\hline $\mathrm{T} 8$ & $60.76 a-c A$ & $50.63 \mathrm{~d}-\mathrm{gB}$ & 55.70 & $16.35 \mathrm{~b}-\mathrm{dA}$ & $14.75 \mathrm{c}-\mathrm{eA}$ & 15.55 & $42.60 \mathrm{c}-\mathrm{gA}$ & $44.17 \mathrm{c}-\mathrm{fA}$ & 43.39 & $13.65 \mathrm{~b}-\mathrm{dA}$ & $15.41 \mathrm{abA}$ & 14.53 \\
\hline T9 & $62.50 \mathrm{~b}-\mathrm{dA}$ & $59.24 \mathrm{~b}-\mathrm{fA}$ & 60.87 & $18.73 \mathrm{abA}$ & $13.51 \mathrm{e}-\mathrm{gB}$ & 16.12 & $44.66 \mathrm{c}-\mathrm{eA}$ & $43.88 \mathrm{c}-\mathrm{eA}$ & 44.27 & $15.28 \mathrm{abA}$ & $13.97 \mathrm{~b}-\mathrm{dA}$ & 14.63 \\
\hline Mean (G) & 57.34 & 54.53 & ---- & 16.25 & 15.11 & ---- & 44.80 & 42.09 & --- & 15.14 & 14.20 & --- \\
\hline \multicolumn{13}{|l|}{ L.S.D. $(0.05)^{*}$} \\
\hline G & \multicolumn{3}{|c|}{ N.S } & \multicolumn{3}{|c|}{ N.S } & \multicolumn{4}{|c|}{ N.S } & \multicolumn{2}{|c|}{ N.S } \\
\hline $\mathrm{T}$ & & 4.39 & & & 1.81 & & & & & & & \\
\hline GX T & & 6.21 & & & 2.56 & & & & & & & \\
\hline
\end{tabular}

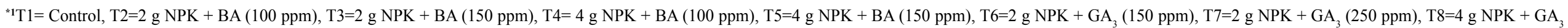

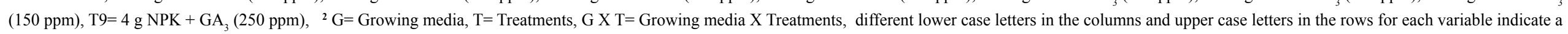
significant difference at $5 \%$ level of significance by LSD test. 
Table 4. Effect of growing media, combined NPK with foliar application of plant growth regulators treatments and the interactions between the two factors on fresh and dry weights of roots of Dracaena marginata during the 2018 and 2019 seasons.

\begin{tabular}{|c|c|c|c|c|c|c|}
\hline \multirow{3}{*}{ Treatments $(T)^{1}$} & \multicolumn{6}{|c|}{$1^{\text {st }}$ season } \\
\hline & \multicolumn{6}{|c|}{ Growing media (G) } \\
\hline & Peat & Peat+ sand & Mean $(T)$ & Peat & Peat + sand & Mean $(\mathrm{T})$ \\
\hline & \multicolumn{3}{|c|}{ F.W of roots } & \multicolumn{3}{|c|}{ D.W of roots } \\
\hline $\mathrm{T} 1$ (Control) & $39.18 \mathrm{jA}$ & $32.90 \mathrm{kB}$ & 36.04 & $14.32 \mathrm{~d}-\mathrm{gA}$ & $11.94 \mathrm{gA}$ & 13.13 \\
\hline $\mathrm{T} 2$ & 48.96 e-gA & $43.00 \mathrm{iB}$ & 45.98 & $14.20 \mathrm{~d}-\mathrm{gA}$ & $15.70 \mathrm{c}-\mathrm{fA}$ & 14.95 \\
\hline $\mathrm{T} 3$ & $50.65 \mathrm{c}-\mathrm{eA}$ & $44.49 \mathrm{hiB}$ & 47.57 & $18.32 \mathrm{bcA}$ & $14.53 \mathrm{~d}-\mathrm{gA}$ & 16.43 \\
\hline $\mathrm{T} 4$ & $50.12 \mathrm{c}-\mathrm{eA}$ & $40.98 \mathrm{jB}$ & 45.55 & $19.06 \mathrm{abA}$ & $17.32 \mathrm{~b}-\mathrm{dA}$ & 18.19 \\
\hline T5 & $46.69 \mathrm{ghA}$ & 44.70 hiA & 45.70 & $14.08 \mathrm{~d}-\mathrm{gB}$ & $19.01 \mathrm{abA}$ & 16.55 \\
\hline T6 & $51.62 \mathrm{c}-\mathrm{eA}$ & $47.08 \mathrm{f}-\mathrm{hB}$ & 49.35 & $16.06 \mathrm{~b}-\mathrm{eA}$ & $14.29 \mathrm{~d}-\mathrm{gA}$ & 15.18 \\
\hline $\mathrm{T} 7$ & $58.76 \mathrm{aA}$ & $54.73 \mathrm{abB}$ & 56.75 & $21.55 \mathrm{aA}$ & $17.28 \mathrm{~b}-\mathrm{dB}$ & 19.42 \\
\hline $\mathrm{T} 8$ & $52.67 \mathrm{cdA}$ & $45.52 \mathrm{hiB}$ & 49.10 & $16.27 \mathrm{~b}-\mathrm{eA}$ & $13.97 \mathrm{e}-\mathrm{gA}$ & 15.12 \\
\hline T9 & $53.42 \mathrm{bcA}$ & $44.30 \mathrm{hiB}$ & 48.86 & $16.23 \mathrm{~b}-\mathrm{eA}$ & 13.93 e-fA & 15.08 \\
\hline Mean $(\mathrm{G})$ & 50.23 & 44.19 & ---- & 16.68 & 15.33 & ---- \\
\hline \multicolumn{7}{|l|}{ L.S.D. $(0.05)^{2}$} \\
\hline G & \multicolumn{3}{|c|}{ Sig. } & \multicolumn{3}{|c|}{ N.S } \\
\hline $\mathrm{T}$ & \multicolumn{3}{|c|}{2.01} & \multicolumn{3}{|c|}{1.97} \\
\hline GX T & \multicolumn{3}{|c|}{2.84} & \multicolumn{3}{|c|}{2.78} \\
\hline \multicolumn{7}{|c|}{$2^{\text {nd }}$ season } \\
\hline T1 (Control) & $32.61 \mathrm{kA}$ & $31.72 \mathrm{kA}$ & 32.17 & $11.29 \mathrm{gA}$ & $10.40 \mathrm{gA}$ & 10.85 \\
\hline $\mathrm{T} 2$ & $50.23 \mathrm{fgA}$ & $46.44 \mathrm{hiB}$ & 48.34 & $15.46 \mathrm{efA}$ & $17.60 \mathrm{~b}-\mathrm{fA}$ & 16.53 \\
\hline $\mathrm{T} 3$ & $52.48 \mathrm{de} A$ & $45.99 \mathrm{hiB}$ & 49.24 & $16.87 \mathrm{c}-\mathrm{fA}$ & $15.76 \mathrm{~d}-\mathrm{fA}$ & 16.32 \\
\hline $\mathrm{T} 4$ & $55.16 \mathrm{cA}$ & $40.32 \mathrm{hB}$ & 47.74 & $17.80 \mathrm{~b}-\mathrm{fA}$ & 20.14 a-cA & 18.97 \\
\hline T5 & $54.27 \mathrm{cA}$ & $42.72 \mathrm{jB}$ & 48.50 & $19.55 \mathrm{a}-\mathrm{cA}$ & $20.46 \mathrm{a}-\mathrm{cA}$ & 20.01 \\
\hline T6 & $55.55 \mathrm{cA}$ & $48.38 \mathrm{ghB}$ & 51.97 & $19.46 \mathrm{a}-\mathrm{dA}$ & $18.94 \mathrm{a}-\mathrm{eA}$ & 19.20 \\
\hline $\mathrm{T} 7$ & $61.80 \mathrm{aA}$ & $51.32 \mathrm{efB}$ & 56.56 & $21.97 \mathrm{aA}$ & $20.97 \mathrm{abA}$ & 21.47 \\
\hline $\mathrm{T} 8$ & $53.53 \mathrm{c}-\mathrm{cA}$ & $46.52 \mathrm{hiB}$ & 50.03 & 19.55 a-cA & $18.19 \mathrm{~b}-\mathrm{fA}$ & 18.87 \\
\hline T9 & $58.80 \mathrm{bA}$ & $46.77 \mathrm{hB}$ & 52.79 & $21.67 \mathrm{abA}$ & $14.92 \mathrm{fB}$ & 18.30 \\
\hline Mean (G) & 52.71 & 44.46 & ---- & 18.18 & 17.49 & ---- \\
\hline \multicolumn{7}{|l|}{ L.S.D. (0.05) * } \\
\hline G & \multicolumn{3}{|c|}{ Sig. } & \multicolumn{3}{|c|}{ Sig. } \\
\hline $\mathrm{T}$ & \multicolumn{3}{|c|}{1.51} & \multicolumn{3}{|c|}{2.28} \\
\hline GX T & \multicolumn{3}{|c|}{2.13} & \multicolumn{3}{|c|}{3.22} \\
\hline
\end{tabular}

${ }^{1} \mathrm{~T} 1=$ Control, $\mathrm{T} 2=2 \mathrm{~g}$ NPK $+\mathrm{BA}(100 \mathrm{ppm}), \mathrm{T} 3=2 \mathrm{~g} \mathrm{NPK}+\mathrm{BA}(150 \mathrm{ppm}), \mathrm{T} 4=4 \mathrm{~g}$ NPK $+\mathrm{BA}(100 \mathrm{ppm}), \mathrm{T} 5=4 \mathrm{~g}$ NPK $+\mathrm{BA}(150 \mathrm{ppm}), \mathrm{T} 6=2 \mathrm{~g}$ $\mathrm{NPK}+\mathrm{GA}_{3}(150 \mathrm{ppm}), \mathrm{T} 7=2 \mathrm{~g} \mathrm{NPK}+\mathrm{GA}_{3}(250 \mathrm{ppm}), \quad \mathrm{T} 8=4 \mathrm{~g} \mathrm{NPK}+\mathrm{GA}_{3}(150 \mathrm{ppm}), \mathrm{T} 9=4 \mathrm{~g} \mathrm{NPK}+\mathrm{GA}_{3}(250 \mathrm{ppm}), \quad{ }^{2} \mathrm{G}=\mathrm{Growing}$ media,

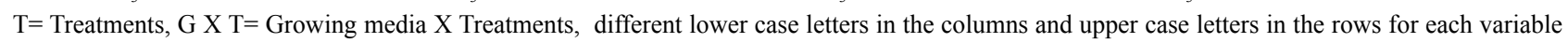
indicate a significant difference at $5 \%$ level of significance by LSD test.. 
The favorable effect of peat- moss medium on enhancing vegetative growth may be attributed to its properties such as low $\mathrm{pH}$ and $\mathrm{EC}$ values in addition to its high water-holing capacity as indicated in (Table 1), this may be associated with water supply in sufficient quantities for turgidity and various positive metabolism including cells enlargement which in turn lead to stimulation of stem elongation (Fascella, 2015; Badran et al., 2017).

\section{Effect of NPK with plant growth regulators treatments}

Data in Tables (2-4) indicated that, the combined treatment of NPK with either BA or $\mathrm{GA}_{3}$ concentrations resulted in significant increases in most of the vegetative growth parameters in terms of plant height, number of leaves/plant, leaf area, root length, fresh and dry weights of leaves, stems and roots as compared to the untreated plants. In spite of this, it was notable that, the only parameter which insignificantly affected by integrated NPK rates with BA or $\mathrm{GA}_{3}$ was stem diameter as compared to each other and control plants. Also, it was detected that, $\mathrm{GA}_{3}$ was more effective than BA when they were combined with NPK at the same rates. These results are in agreement with the finding of the previous study on croton plants (Ibrahim et al., 2010) who reported that $\mathrm{GA}_{3}$ treatments were more effective than BA on increasing plant height, leaf area, fresh and dry weights of stems, leaves and roots of croton plants.

Concerning the application NPK combined with the same concentrations of plant growth regulators (BA or $\mathrm{GA}_{3}$ ), it was found that the lowest rate of NPK was more effective than the highest one; this may be due to plant sensitively against salt toxicity resulting from excessive fertilization rates. In both seasons, the highest values for most vegetative parameters were obtained from the plants treated with the combination of NPK at $2 \mathrm{~g} \mathrm{pot}^{-1}$ with $\mathrm{GA}_{3}$ at $250 \mathrm{ppm}$, while the lowest values were resulted from the control plants. Similar increases in the vegetative growth parameters due to application of NPK in combination with $\mathrm{GA}_{3}$ had been authenticated by previous research (El-Sayed et al., 2016). Also, increases in growth parameters of Dendrobium densiflorum plants as a result of combined NPK with BA have been reported by prior study (Ngapui et al., 2018).

The above-mentioned results showed the important role of $\mathrm{N}, \mathrm{P}$ and $\mathrm{K}$ as well as $\mathrm{BA}$ and $\mathrm{GA}_{3}$ in the different physiological processes within the plant, which in turn affect the plant growth. Nitrogen is an important macroelement in organic molecules in plants including proteins, nucleic acids, purines, pyrimidines, co-enzymes (vitamins), and production of chlorophyll. Phosphorus is a component of sugar phosphates, nucleic acids, nucleotides, coenzymes, phospholipids, however potassium is essential in activation of enzymes and co-enzymes, proteins formation, photosynthesis, sugar transport, the uptake of other nutrients and their movement within the plant. The stimulated effect of BA may be due to its role in increasing cell division and enlargement, branches formation and overcoming the apical dominance leading to increase plant growth. The favorable effect of $\mathrm{GA}_{3}$ on the plant growth may be attributed to its effect on stimulating the expression of enzymes involved in cell wall loosening and genes controlling cell division and also stimulating microtubule rearrangements associated with cell expansion (Satish and Manju, 2018). It was stated that the increase of plant growth by BA mainly associated with higher net assimilation rates and increased $\mathrm{N}$ content per unit leaf area (Di Benedetto and Galmarini, 2015), while increasing plant growth due to $\mathrm{GA}_{3}$ may be attributed to its influence on increasing photosynthesis rate through increasing leaf surface (Lima et al., 2014). Moreover, increasing plant growth as a result of combined growth hormones with nutrient may be owing to physiological role of hormones and nutrient in synthesis of the plant phytochemicals through the action of various enzymes activity and protein synthesis (Tandel et al., 2018).

Effect of the interaction between growing media and NPK combined with plant growth regulators treatments

Data presented in Tables (2-4) revealed that generally, the plants grown in peat- moss or peat-moss + sand and received different NPK combined with $\mathrm{BA}$ and $\mathrm{GA}_{3}$ treatments were significantly higher than those of the control plants. In both seasons, in most cases, the highest values recorded for most of the vegetative characteristics were obtained from plants grown in peat moss medium and received of $2 \mathrm{~g} \mathrm{NPK}+\mathrm{GA}_{3}$ at $250 \mathrm{ppm}$ as compared with the control which giving lowest values.

\section{Chemical constituents}

\section{Total chlorophylls and total carbohydrates contents}

From the data Table (5) it's possibly discerned that, in both seasons, total chlorophyll in the leaves and total carbohydrates in the leaves and stems of Dracaena marginata 'Bicolor' were significantly higher in the plants grown in peat-moss + sand medium than those grown in peat-moss alone. These findings are in accordance with the results obtained by previous studies which showed that a mixture peat-moss with sand increased chlorophyll content (EL-Quasni et al., 2014; Abd el Gayed and Attia, 2018). 
Table 5: Effect of growing media, combined NPK with foliar application of plant growth regulators treatments and the interactions between the two factors on total chlorophylls, total carbohydrates in leaves and stems of Dracaena marginata during the 2018 and 2019 seasons.

\begin{tabular}{|c|c|c|c|c|c|c|c|c|c|}
\hline \multirow{4}{*}{$\begin{array}{l}\text { Treatments } \\
\text { (T) }{ }^{1}\end{array}$} & \multicolumn{9}{|c|}{$1^{\text {st }}$ season } \\
\hline & \multicolumn{9}{|c|}{ Growing media (G) } \\
\hline & Peat & Peat+ sand & Mean $(\mathrm{T})$ & Peat & Peat + sand & Mean $(\mathrm{T})$ & Peat & Peat+ sand & Mean $(\mathrm{T})$ \\
\hline & \multicolumn{3}{|c|}{$\begin{array}{c}\text { Total chlorophylls (SPAD) } \\
\text { in leaves }\end{array}$} & \multicolumn{3}{|c|}{ Total carbohydrates $(\%)$ in leaves } & \multicolumn{3}{|c|}{ Total carbohydrates $(\%)$ in stem } \\
\hline T1 (Control) & $54.50 \mathrm{iB}$ & $63.10 \mathrm{ghA}$ & 58.80 & $35.11 \mathrm{LA}$ & $33.18 \mathrm{mB}$ & 34.15 & $29.25 \mathrm{hA}$ & $27.93 \mathrm{hA}$ & 28.59 \\
\hline $\mathrm{T} 2$ & $60.33 \mathrm{hB}$ & $64.30 \mathrm{gA}$ & 62.32 & $37.97 \mathrm{jkA}$ & $36.911 \mathrm{~A}$ & 37.44 & $33.27 \mathrm{gA}$ & $31.21 \mathrm{gB}$ & 32.24 \\
\hline $\mathrm{T} 3$ & $72.43 \mathrm{~b}-\mathrm{dB}$ & $75.96 \mathrm{abA}$ & 74.20 & $39.35 \mathrm{ijA}$ & $38.25 \mathrm{jkA}$ & 38.80 & 36.11 efA & $32.19 \mathrm{gB}$ & 34.15 \\
\hline $\mathrm{T} 4$ & 68.10 efB & $73.00 \mathrm{~b}-\mathrm{dA}$ & 70.55 & $37.21 \mathrm{kB}$ & $40.21 \mathrm{hiA}$ & 38.71 & $35.12 \mathrm{fB}$ & 36.52 d-fA & 35.82 \\
\hline $\mathrm{T} 5$ & 67.10 efA & $69.53 \mathrm{deA}$ & 68.32 & $41.65 \mathrm{ghB}$ & $44.60 \mathrm{eA}$ & 43.13 & $\begin{array}{l}37.29 \\
\mathrm{c}-\mathrm{eB}\end{array}$ & $38.22 \mathrm{cA}$ & 37.75 \\
\hline T6 & 64.53 efB & 68.13 efA & 66.33 & $40.95 \mathrm{hiB}$ & $42.85 \mathrm{fgA}$ & 41.90 & $35.25 \mathrm{fA}$ & $36.55 \mathrm{~d}-\mathrm{fA}$ & 35.90 \\
\hline $\mathrm{T} 7$ & $69.40 \mathrm{deB}$ & $78.10 \mathrm{aA}$ & 73.75 & $39.96 \mathrm{hiB}$ & 43.96 efA & 41.96 & $37.76 \mathrm{cdB}$ & $39.86 \mathrm{bA}$ & 38.81 \\
\hline $\mathrm{T} 8$ & $70.76 \mathrm{c}-\mathrm{eB}$ & $74.40 \mathrm{bcA}$ & 72.58 & $48.37 \mathrm{cA}$ & $46.31 \mathrm{~dB}$ & 47.34 & $39.88 \mathrm{bA}$ & $40.18 \mathrm{bA}$ & 40.03 \\
\hline T9 & $72.00 \mathrm{cdB}$ & $71.73 \mathrm{c}-\mathrm{eA}$ & 71.87 & $55.82 \mathrm{bB}$ & $58.61 \mathrm{aA}$ & 57.22 & $40.21 \mathrm{bB}$ & $43.11 \mathrm{aA}$ & 41.66 \\
\hline Mean $(G)$ & 66.57 & 70.92 & ---- & 41.82 & 42.76 & ---- & 36.02 & 36.20 & ---- \\
\hline \multicolumn{10}{|l|}{$\begin{array}{l}\text { L.S.D. } \\
(0.05)^{2}\end{array}$} \\
\hline G & \multicolumn{3}{|c|}{ Sig. } & \multicolumn{3}{|c|}{ Sig. } & \multicolumn{3}{|c|}{ Sig. } \\
\hline $\mathrm{T}$ & \multicolumn{3}{|c|}{2.40} & \multicolumn{3}{|c|}{1.14} & \multicolumn{3}{|c|}{0.99} \\
\hline GX T & \multicolumn{3}{|c|}{3.39} & \multicolumn{3}{|c|}{1.61} & \multicolumn{3}{|c|}{1.40} \\
\hline \multicolumn{10}{|c|}{$2^{\text {nd }}$ season } \\
\hline T1 (Control) & $59.30 \mathrm{hA}$ & $62.93 \mathrm{ghA}$ & 61.12 & $\begin{array}{l}32.16 \\
\operatorname{lm} A\end{array}$ & $\begin{array}{l}31.63 \\
\mathrm{k}-\mathrm{mA}\end{array}$ & 31.90 & $26.40 \mathrm{lB}$ & $29.11 \mathrm{kA}$ & 27.76 \\
\hline $\mathrm{T} 2$ & $65.43 \mathrm{fgA}$ & $65.90 \mathrm{e}-\mathrm{gA}$ & 65.67 & $34.87 \mathrm{jkA}$ & $34.52 \mathrm{jkA}$ & 34.70 & $31.52 \mathrm{jB}$ & $34.17 \mathrm{hiA}$ & 32.85 \\
\hline $\mathrm{T} 3$ & $70.40 \mathrm{~b}-\mathrm{eB}$ & $78.06 \mathrm{aA}$ & 74.23 & $33.21 \mathrm{klB}$ & $39.75 \mathrm{fA}$ & 36.48 & $33.44 \mathrm{hiA}$ & $32.74 \mathrm{ijA}$ & 33.09 \\
\hline $\mathrm{T} 4$ & $70.66 \mathrm{~b}-\mathrm{dA}$ & $73.93 \mathrm{abA}$ & 72.30 & $36.17 \mathrm{ijA}$ & $37.11 \mathrm{hiA}$ & 36.64 & $36.31 \mathrm{deA}$ & $32.91 \mathrm{iB}$ & 34.61 \\
\hline $\mathrm{T} 5$ & $71.46 \mathrm{~b}-\mathrm{dA}$ & $72.40 \mathrm{~b}-\mathrm{dA}$ & 71.93 & $\begin{array}{l}37.54 \\
\mathrm{~g}-\mathrm{iB}\end{array}$ & $39.56 \mathrm{fA}$ & 38.55 & $34.49 \mathrm{f}-\mathrm{hA}$ & $33.82 \mathrm{~g}-\mathrm{iA}$ & 34.16 \\
\hline T6 & $67.73 \mathrm{~d}-\mathrm{fA}$ & $71.90 \mathrm{~b}-\mathrm{dA}$ & 69.82 & $41.77 \mathrm{deA}$ & $39.27 \mathrm{fgB}$ & 40.52 & $37.90 \mathrm{bcA}$ & $34.59 \mathrm{f}-\mathrm{hB}$ & 36.25 \\
\hline $\mathrm{T} 7$ & $70.93 \mathrm{~b}-\mathrm{dB}$ & $78.30 \mathrm{aA}$ & 74.62 & $42.94 \mathrm{dA}$ & $38.42 \mathrm{f}-\mathrm{hB}$ & 40.68 & $37.11 \mathrm{cdA}$ & $36.96 \mathrm{cdA}$ & 37.04 \\
\hline $\mathrm{T} 8$ & $68.86 \mathrm{c}-\mathrm{fB}$ & $74.96 \mathrm{abA}$ & 71.91 & $40.28 \mathrm{efB}$ & $46.86 \mathrm{cA}$ & 43.57 & $35.23 \mathrm{e}-\mathrm{gB}$ & $38.59 \mathrm{bA}$ & 36.91 \\
\hline T9 & $72.53 \mathrm{~b}-\mathrm{dA}$ & $73.13 \mathrm{bcA}$ & 72.83 & $48.88 \mathrm{bB}$ & $53.57 \mathrm{aA}$ & 51.23 & 35.39 efB & $39.95 \mathrm{aA}$ & 37.67 \\
\hline Mean (G) & 68.59 & 72.39 & --- & 38.65 & 40.08 & ---- & 34.20 & 34.76 & ---- \\
\hline \multicolumn{10}{|l|}{$\begin{array}{l}\text { L.S.D. } \\
(0.05)^{*}\end{array}$} \\
\hline G & \multicolumn{3}{|c|}{ Sig. } & \multicolumn{3}{|c|}{ Sig. } & \multicolumn{3}{|c|}{ Sig. } \\
\hline $\mathrm{T}$ & \multicolumn{3}{|c|}{2.99} & \multicolumn{3}{|c|}{1.27} & \multicolumn{3}{|c|}{0.93} \\
\hline GX T & \multicolumn{3}{|c|}{4.23} & \multicolumn{3}{|c|}{1.79} & \multicolumn{3}{|c|}{1.32} \\
\hline
\end{tabular}

${ }^{1} \mathrm{~T} 1=$ Control, $\mathrm{T} 2=2 \mathrm{~g}$ NPK $+\mathrm{BA}(100 \mathrm{ppm}), \mathrm{T} 3=2 \mathrm{~g} \mathrm{NPK}+\mathrm{BA}(150 \mathrm{ppm}), \mathrm{T} 4=4 \mathrm{~g}$ NPK $+\mathrm{BA}(100 \mathrm{ppm}), \mathrm{T} 5=4 \mathrm{~g}$ NPK $+\mathrm{BA}(150 \mathrm{ppm}), \mathrm{T} 6=2 \mathrm{~g}$ $\mathrm{NPK}+\mathrm{GA}_{3}(150 \mathrm{ppm}), \mathrm{T} 7=2 \mathrm{~g} \mathrm{NPK}+\mathrm{GA}_{3}(250 \mathrm{ppm}), \quad \mathrm{T} 8=4 \mathrm{~g} \mathrm{NPK}+\mathrm{GA}_{3}(150 \mathrm{ppm}), \mathrm{T} 9=4 \mathrm{~g} \mathrm{NPK}+\mathrm{GA}_{3}(250 \mathrm{ppm}),{ }^{2} \mathrm{G}=\mathrm{Growing}$ media, $\mathrm{T}=$ Treatments, G X T= Growing media X Treatments, different lower case letters in the columns and upper case letters in the rows for each variable indicate a significant difference at $5 \%$ level of significance by LSD test. 
The data in the data Table (5) also showed that application of the combined NPK with either BA or $\mathrm{GA}_{3}$ significantly increased total chlorophyll content in the leaves and total carbohydrates in leaves and stems as compared to the untreated plants. Similar results on increasing the contents of chlorophylls or carbohydrates due to application the combined NPK with $\mathrm{GA}_{3}$ were reported by previous study (El-Sayed et al., 2016; Ghatas, 2016). Earlier studies (Tandel et al., 2018) reported increase in carbohydrates content due to combined urea with BA.

In both seasons, the lowest values of total chlorophyll content were obtained from plants grown in peat-moss medium without additional treatments, while the highest values were obtained from the plants grown in peat-moss + sand and received $2 \mathrm{~g} \mathrm{NPK}+\mathrm{GA}_{3}$ at $250 \mathrm{ppm}$. In both seasons, the highest values of total carbohydrates were recorded in the plants grown in peat moss + sand and received $4 \mathrm{~g} \mathrm{NPK}+\mathrm{GA}_{3}$ at $250 \mathrm{ppm}$, whereas the lowest values were determined in the plants grown in peat-moss + sand without treatments.

\section{Nutrients content:}

\section{Macro elements}

The chemical analysis of the leaves and stems (Tables 6 and 7) demonstrated that, generally, the uptake and accumulation of macro elements represented in $\mathrm{N}, \mathrm{P}, \mathrm{K}$, $\mathrm{Ca}$ and $\mathrm{Mg} \%$ were significantly higher in peat moss-grown plants than those grown in peat-moss + sand. However, in the first season there were no significant differences between the two tested growing media concerning the values of $\mathrm{P}$ and $\mathrm{Mg} \%$ in stems and $\mathrm{K} \%$ in the leaves.

The obtained results of increased $\mathrm{N}, \mathrm{P}$ or $\mathrm{K} \%$ in plants grown in peat moss alone compared to other media are similar to those found by prior research (Mousa et al., 2015). The obtained results of increased the accumulation of macro elements in peat moss-grown plants could be explained on the basis of high content of organic matter in peat moss maybe increase the activity of the beneficial microorganisms that play vital role in soil aeration and availability of the elements in rhizosphere to be absorbed by the plant roots. Moreover, its proper $\mathrm{pH}$ and EC values, high water-holing capacity may be had favorable effect in availability of nutrients and its accumulation in plant organs (Abdul-Hafeez et al., 2015; Pascual et al., 2018).

As for the effect of NPK in combination with BA and $\mathrm{GA}_{3}$ the data in Tables ( 6 and 7$)$ pointed out that, application of the combined treatments resulted in significant increases in $\mathrm{N}, \mathrm{P}, \mathrm{K}, \mathrm{Ca}$ and $\mathrm{Mg} \%$ in the leaves and stems compared to the control plants. However, in the first season plants treated with the combined treatments of $2 \mathrm{~g} \mathrm{NPK}+\mathrm{BA}$ at 100 or $150 \mathrm{ppm}$ (T1 and T2) had insignificantly higher values of $\mathrm{P} \%$ in the stems than the control plants. The data also showed that, in most cases, the values of macro nutrients were increased steadily with increasing application rate of NPK and BA or $\mathrm{GA}_{3}$ concentration compared the control plants. Furthermore, the combined NPK with $\mathrm{GA}_{3}$ appeared to be more effective than combined with BA at the high concentration (250 ppm). These results of increased N, P or $\mathrm{K} \%$ as a result of accompanied NPK with $\mathrm{GA}_{3}$ treatments are in agreement with those obtained by prior research (ElSayed et al., 2016; Ghatas, 2016), while increased N, P or $\mathrm{K} \%$ due to the combined NPK with BA treatments are in harmony with prior study (Barman and Naik, 2017).

Regarding the interaction effect, data in Tables (6 and 7) showed that, plants grown in peat moss only or peat moss + sand and received different combined treatments of NPK with either BA or $\mathrm{GA}_{3}$ resulted in significant increment of mineral nutrients percentage than those resulted from control plants. The data also revealed that, under both growing media $\mathrm{GA}_{3}$ was more effective than $\mathrm{BA}$ when combined with NPK at the same rate.

\section{Micro elements}

The data in in Tables ( 8 and 9) showed that in most cases, the values of micro elements represented in $\mathrm{Cu}, \mathrm{Fe}, \mathrm{Mn}, \mathrm{Zn}$ and $\mathrm{B}$ in the leaves and stems of plants grown in peat-moss + sand were higher than those of the plants grown in peatmoss alone. In the first season the accumulation of $\mathrm{Zn}$ and $\mathrm{B}$ in stems was the only exceptions which showed different trend, as peat moss grown plants produced significantly higher values than peat moss + sand medium. 


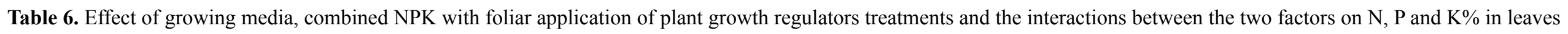
and stems of Dracaena marginata during the 2018 and 2019 seasons.

\begin{tabular}{|c|c|c|c|c|c|c|c|c|c|c|c|c|c|c|c|c|c|c|}
\hline \multirow{4}{*}{$\begin{array}{l}\text { Treatments } \\
\text { (T) }\end{array}$} & \multicolumn{18}{|c|}{$1^{\text {st }}$ season } \\
\hline & \multicolumn{18}{|c|}{ Growing media (G) } \\
\hline & Peat & Peat+ sand & Mean $(\mathrm{T})$ & Peat & Peat + sand & $\begin{array}{l}\text { Mean } \\
(\mathrm{T})\end{array}$ & Peat & Peat + sand & Mean $(T)$ & Peat & Peat+ sand & $\begin{array}{l}\text { Mean } \\
(\mathrm{T})\end{array}$ & Peat & Peat + sand & Mean $(T)$ & Peat & Peat + sand & Mean $(\mathrm{T})$ \\
\hline & \multicolumn{3}{|c|}{$\mathbf{N}[\%]$ in leaves } & \multicolumn{3}{|c|}{$\mathbf{N}[\%]$ in stems } & \multicolumn{3}{|c|}{$P[\%]$ in leaves } & \multicolumn{3}{|c|}{$\mathbf{P}[\%]$ in stems } & \multicolumn{3}{|c|}{$\mathbf{K}[\%]$ in leaves } & \multicolumn{3}{|c|}{$\mathrm{K}[\%]$ in stems } \\
\hline T1 (Control) & $1.54 \mathrm{cA}$ & $1.56 \mathrm{cA}$ & 1.55 & $0.97 \mathrm{iA}$ & $1.02 \mathrm{iA}$ & 1.00 & $0.22 \mathrm{gA}$ & $0.24 \mathrm{fgA}$ & 0.23 & 0.18 & 0.20 & $0.19 \mathrm{a}$ & $2.02 \mathrm{gB}$ & $2.11 \mathrm{hA}$ & 2.07 & $1.98 \mathrm{iA}$ & $2.00 \mathrm{iA}$ & 1.99 \\
\hline $\mathrm{T} 2$ & $1.77 \mathrm{bcA}$ & $1.69 \mathrm{bcA}$ & 1.73 & $1.47 \mathrm{ghA}$ & $1.45 \mathrm{hA}$ & 1.46 & $0.29 \mathrm{c}-\mathrm{fA}$ & $0.27 \mathrm{e}-\mathrm{gA}$ & 0.28 & 0.20 & 0.21 & $0.21 \mathrm{a}$ & $2.27 \mathrm{fA}$ & $2.22 \mathrm{hA}$ & 2.25 & $2.07 \mathrm{hA}$ & $2.09 \mathrm{ghA}$ & 2.08 \\
\hline $\mathrm{T} 3$ & $1.83 \mathrm{a}-\mathrm{cA}$ & $1.78 \mathrm{bcA}$ & 1.81 & $1.53 \mathrm{efA}$ & $1.49 \mathrm{f}-\mathrm{hA}$ & 1.51 & $0.28 \mathrm{~d}-\mathrm{fA}$ & $0.29 \mathrm{c}-\mathrm{fA}$ & $0.29 b$ & 0.22 & 0.23 & $0.23 \mathrm{a}$ & $2.43 \mathrm{dA}$ & $2.39 \mathrm{dfA}$ & 2.41 & $2.10 \mathrm{ghA}$ & $2.13 \mathrm{fgA}$ & 2.12 \\
\hline $\mathrm{T} 4$ & $1.92 \mathrm{a}-\mathrm{cA}$ & $1.87 \mathrm{a}-\mathrm{cA}$ & 1.90 & $1.57 \mathrm{deA}$ & 1.53 efA & 1.55 & $0.30 \mathrm{~b}-\mathrm{fA}$ & $0.29 \mathrm{c}-\mathrm{f} A$ & $0.30 \mathrm{~b}$ & 0.24 & 0.24 & $0.24 d$ & $2.49 \mathrm{cA}$ & $2.42 \mathrm{dfB}$ & 2.46 & $2.17 \mathrm{~d}$-fA & $2.15 \mathrm{egA}$ & 2.16 \\
\hline T5 & $2.17 \mathrm{a}-\mathrm{cA}$ & $2.23 \mathrm{a}-\mathrm{cA}$ & 2.20 & $1.63 \mathrm{bcA}$ & $1.61 \mathrm{cdA}$ & 1.62 & $0.33 \mathrm{~b}-\mathrm{dA}$ & $0.31 \mathrm{~b}-\mathrm{eAA}$ & 0.32 & 0.25 & 0.26 & $0.26 \mathrm{c}$ & $2.51 \mathrm{cA}$ & $2.49 \mathrm{cA}$ & 2.50 & $2.26 \mathrm{bA}$ & $2.24 \mathrm{bcA}$ & 2.25 \\
\hline T6 & $2.21 \mathrm{a}-\mathrm{cB}$ & $2.33 \mathrm{a}-\mathrm{cA}$ & 2.27 & $1.52 \mathrm{e}-\mathrm{gA}$ & $1.48 \mathrm{f}-\mathrm{hA}$ & 1.50 & $0.30 \mathrm{~b}$-fA & $0.32 \mathrm{~b}-\mathrm{eA}$ & 0.31 & 0.27 & 0.26 & $0.27 \mathrm{~b}$ & $2.31 \mathrm{fB}$ & $2.37 \mathrm{eA}$ & 2.34 & $2.13 \mathrm{fgA}$ & $2.11 \mathrm{ghA}$ & 2.12 \\
\hline $\mathrm{T} 7$ & $2.42 \mathrm{a}-\mathrm{cA}$ & $2.37 \mathrm{a}-\mathrm{cA}$ & 2.40 & $1.61 \mathrm{cdA}$ & $1.57 \mathrm{deA}$ & 1.59 & $0.32 \mathrm{~b}-\mathrm{eA}$ & $0.30 \mathrm{~b}$-fA & 0.31 & 0.28 & 0.27 & $0.28 \mathrm{a}$ & $2.54 \mathrm{cA}$ & $2.49 \mathrm{cA}$ & 2.52 & $2.19 \mathrm{c}-\mathrm{eA}$ & $2.17 \mathrm{~d}-\mathrm{fA}$ & 2.18 \\
\hline $\mathrm{T} 8$ & $2.57 \mathrm{a}-\mathrm{cA}$ & $2.52 \mathrm{a}-\mathrm{cA}$ & 2.55 & $1.64 \mathrm{bcA}$ & $1.60 \mathrm{cdA}$ & 1.62 & $0.36 \mathrm{bA}$ & $0.30 \mathrm{~b}-\mathrm{fB}$ & 0.33 & 0.29 & 0.26 & $0.28 \mathrm{a}$ & $2.79 \mathrm{bA}$ & $2.80 \mathrm{bA}$ & 2.80 & $2.25 \mathrm{bA}$ & $2.22 \mathrm{~b}-\mathrm{dA}$ & 2.24 \\
\hline T9 & $2.87 \mathrm{aA}$ & $2.70 \mathrm{abB}$ & 2.79 & $1.74 \mathrm{aA}$ & $1.67 \mathrm{bA}$ & 1.71 & $0.39 \mathrm{aA}$ & $0.33 b-d B$ & 0.36 & 0.30 & 0.26 & $0.28 \mathrm{a}$ & $3.21 \mathrm{aA}$ & $3.19 \mathrm{aA}$ & 3.20 & $2.39 \mathrm{aA}$ & $2.34 \mathrm{aA}$ & 2.37 \\
\hline Mean (G) & 2.14 & 2.12 & ---- & 1.52 & 1.49 & ---- & 0.31 & 0.29 & ---- & $0.25 \mathrm{~A}$ & $0.24 \mathrm{~B}$ & ---- & 2.51 & 2.50 & ---- & 2.17 & 2.16 & ---- \\
\hline \multicolumn{19}{|l|}{$\begin{array}{l}\text { L.S.D. } \\
(0.05)^{2}\end{array}$} \\
\hline G & \multicolumn{3}{|c|}{ Sig. } & \multicolumn{3}{|c|}{ Sig. } & \multicolumn{3}{|c|}{ Sig. } & \multicolumn{3}{|c|}{ N.S } & \multicolumn{3}{|c|}{ N.S } & \multicolumn{3}{|c|}{ Sig. } \\
\hline $\mathrm{T}$ & \multicolumn{3}{|c|}{0.07} & \multicolumn{3}{|c|}{0.06} & \multirow{2}{*}{\multicolumn{3}{|c|}{0.02}} & \multirow{2}{*}{\multicolumn{3}{|c|}{0.05}} & \multicolumn{3}{|c|}{0.04} & \multicolumn{3}{|c|}{0.04} \\
\hline $\mathrm{GXT}$ & \multicolumn{3}{|c|}{0.10} & & 0.08 & & & & & & & & & 0.06 & & & 0.07 & \\
\hline & & & & & & & & $2^{\text {nd }} s$ & season & & & & & & & & & \\
\hline T1 (Control) & $1.45 \mathrm{iA}$ & $1.48 \mathrm{iA}$ & 1.47 & $1.07 \mathrm{hA}$ & $1.09 \mathrm{hA}$ & 1.08 & $0.25 \mathrm{iB}$ & 0.29 hiA & 0.27 & 0.20 & 0.22 & $0.21 \mathrm{~h}$ & $1.57 \mathrm{jA}$ & $1.59 \mathrm{jA}$ & 1.58 & $1.96 \mathrm{hB}$ & $2.13 \mathrm{gA}$ & 2.05 \\
\hline $\mathrm{T} 2$ & $1.79 \mathrm{ghA}$ & $1.66 \mathrm{hA}$ & 1.73 & $1.59 \mathrm{~d}-\mathrm{fB}$ & $1.39 \mathrm{gA}$ & 1.49 & $0.37 \mathrm{~d}-\mathrm{gA}$ & $0.35 \mathrm{fgA}$ & 0.36 & 0.23 & 0.25 & $0.24 \mathrm{~g}$ & $1.70 \mathrm{iA}$ & $1.58 \mathrm{j} \mathrm{B}$ & 1.64 & $2.25 \mathrm{de} A$ & $2.30 \mathrm{cA}$ & 2.28 \\
\hline $\mathrm{T} 3$ & $1.78 \mathrm{ghA}$ & $1.77 \mathrm{ghA}$ & 1.78 & $1.49 \mathrm{fgA}$ & $1.52 \mathrm{fA}$ & 1.51 & $0.37 \mathrm{~d}-\mathrm{gA}$ & $0.36 \mathrm{f}-\mathrm{gA}$ & 0.37 & 0.27 & 0.25 & $0.26 \mathrm{f}$ & $1.80 \mathrm{hA}$ & $1.78 \mathrm{hA}$ & 1.79 & $2.31 \mathrm{cB}$ & $2.45 \mathrm{bA}$ & 2.38 \\
\hline $\mathrm{T} 4$ & $1.69 \mathrm{hB}$ & $2.02 \mathrm{fA}$ & 1.86 & $1.65 \mathrm{~dB}$ & $1.78 \mathrm{cA}$ & 1.72 & $0.41 \mathrm{c}-\mathrm{eA}$ & $0.33 \mathrm{ghB}$ & 0.37 & 0.32 & 0.29 & $0.31 \mathrm{~d}$ & $1.94 \mathrm{gA}$ & $1.76 \mathrm{hB}$ & 1.85 & $2.52 \mathrm{aA}$ & $2.11 \mathrm{gB}$ & 2.32 \\
\hline T5 & 2.11 efA & $1.94 \mathrm{fgB}$ & 2.03 & $1.66 \mathrm{~dB}$ & $1.89 \mathrm{bA}$ & 1.78 & $0.44 \mathrm{bcA}$ & $0.33 \mathrm{ghB}$ & 0.39 & 0.32 & 0.34 & $0.33 \mathrm{~b}$ & $2.16 \mathrm{dA}$ & $2.00 \mathrm{efB}$ & 2.08 & $2.51 \mathrm{aB}$ & $2.26 \mathrm{c}-\mathrm{eA}$ & 2.39 \\
\hline T6 & $2.27 \mathrm{deA}$ & $2.28 \mathrm{deA}$ & 2.28 & $1.58 \mathrm{~d}-\mathrm{fA}$ & $1.51 \mathrm{fA}$ & 1.55 & $0.41 \mathrm{c}-\mathrm{eA}$ & $0.35 \mathrm{fgB}$ & 0.38 & 0.31 & 0.27 & $0.29 \mathrm{e}$ & $1.95 \mathrm{fg} A$ & $1.77 \mathrm{hB}$ & 1.86 & $2.10 \mathrm{cB}$ & $2.19 \mathrm{fA}$ & 2.15 \\
\hline $\mathrm{T} 7$ & $2.52 \mathrm{bcA}$ & $1.99 \mathrm{fB}$ & 2.26 & $1.79 \mathrm{cA}$ & $1.53 \mathrm{efB}$ & 1.66 & $0.39 \mathrm{c}-\mathrm{fA}$ & $0.32 \mathrm{ghB}$ & 0.36 & 0.35 & 0.28 & $0.32 \mathrm{c}$ & $1.96 \mathrm{gA}$ & $2.04 \mathrm{eA}$ & 2.00 & $2.21 \mathrm{efB}$ & $2.26 \mathrm{c}-\mathrm{eA}$ & 2.24 \\
\hline $\mathrm{T} 8$ & $2.39 \mathrm{cdB}$ & $2.62 \mathrm{bA}$ & 2.51 & $1.59 \mathrm{~d}-\mathrm{fA}$ & $1.68 \mathrm{dA}$ & 1.64 & $0.43 \mathrm{bcA}$ & $0.42 \mathrm{~b}-\mathrm{dA}$ & 0.43 & 0.36 & 0.30 & $0.33 b$ & $2.10 \mathrm{cA}$ & $2.11 \mathrm{cdA}$ & 2.11 & $2.27 \mathrm{c}-\mathrm{dA}$ & $2.24 \mathrm{~d}-\mathrm{fA}$ & 2.26 \\
\hline T9 & $2.97 \mathrm{aA}$ & $2.62 \mathrm{bB}$ & 2.80 & $1.99 \mathrm{aA}$ & $1.63 \mathrm{de}$ & 1.81 & $0.51 \mathrm{aA}$ & $0.47 \mathrm{abB}$ & 0.49 & 0.39 & 0.33 & $0.36 \mathrm{a}$ & $2.44 \mathrm{aA}$ & $2.31 \mathrm{bB}$ & 2.38 & $2.54 \mathrm{aA}$ & $2.41 \mathrm{bB}$ & 2.48 \\
\hline Mean (G) & 2.11 & 2.04 & ---- & 1.60 & $1.56 \mathrm{~B}$ & ---- & 0.40 & 0.36 & ---- & $0.31 \mathrm{~A}$ & $0.28 \mathrm{~B}$ & ---- & 1.96 & 1.88 & ---- & 2.30 & 2.26 & ---- \\
\hline $\begin{array}{l}\text { L.S.D. } \\
(0.05)^{*}\end{array}$ & & & & & & & & & & & & & & & & & & \\
\hline $\mathrm{G}$ & & Sig. & & & Sig. & & & Sig. & & & Sig. & & & Sig. & & & Sig. & \\
\hline $\mathrm{T}$ & & 0.11 & & & 0.08 & & & 0.03 & & & 0.01 & & & 0.06 & & & 0.03 & \\
\hline G X T & & 0.16 & & & 0.11 & & & 0.04 & & & 0.16 & & & 0.09 & & & 0.05 & \\
\hline
\end{tabular}




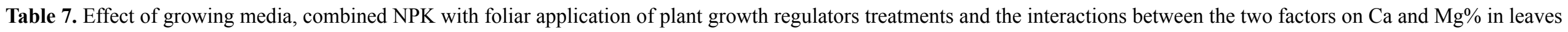
and stems of Dracaena marginata during the 2018 and 2019 seasons.

\begin{tabular}{|c|c|c|c|c|c|c|c|c|c|c|c|c|}
\hline \multirow{3}{*}{ Treatments $(\mathrm{T})^{1}$} & \multicolumn{12}{|c|}{$1^{\text {st }}$ season } \\
\hline & \multicolumn{12}{|c|}{ Growing media (G) } \\
\hline & Peat & Peat+ sand & Mean $(T)$ & Peat & Peat+ sand & Mean $(\mathrm{T})$ & Peat & Peat+ sand & Mean $(\mathrm{T})$ & Peat & Peat+ sand & Mean $(\mathrm{T})$ \\
\hline & \multicolumn{3}{|c|}{ Ca $[\%]$ in leaves } & \multicolumn{3}{|c|}{$\mathrm{Ca}[\%]$ in stems } & \multicolumn{3}{|c|}{$\operatorname{Mg}[\%]$ in leaves } & \multicolumn{3}{|c|}{$\operatorname{Mg}[\%]$ in stems } \\
\hline T1 (Control) & $1.49 \mathrm{hA}$ & $1.50 \mathrm{hA}$ & 1.50 & $1.30 \mathrm{jA}$ & $1.33 \mathrm{~h}-\mathrm{jA}$ & 1.32 & $0.25 \mathrm{ijA}$ & $0.23 \mathrm{jA}$ & 0.24 & $0.21 \mathrm{gA}$ & $0.20 \mathrm{gA}$ & 0.21 \\
\hline $\mathrm{T} 2$ & $2.13 \mathrm{fA}$ & $1.93 \mathrm{gB}$ & 2.03 & $1.43 \mathrm{fA}$ & $1.35 \mathrm{~g}-\mathrm{iB}$ & 1.39 & $0.27 \mathrm{~h}-\mathrm{jA}$ & $0.25 \mathrm{ijA}$ & 0.26 & $0.24 \mathrm{efA}$ & $0.26 \mathrm{~d}-\mathrm{fA}$ & 0.25 \\
\hline $\mathrm{T} 3$ & $2.19 \mathrm{efA}$ & $2.16 \mathrm{fA}$ & 2.18 & $1.36 \mathrm{~g}-\mathrm{iA}$ & $1.38 \mathrm{f}-\mathrm{gA}$ & 1.37 & 0.29hiA & $0.27 \mathrm{~h}-\mathrm{jA}$ & 0.28 & $0.27 \mathrm{~d}-\mathrm{fA}$ & 0.24 efA & 0.26 \\
\hline $\mathrm{T} 4$ & $2.25 \mathrm{de} A$ & $2.21 \mathrm{efA}$ & 2.23 & 1.31ijB & $1.40 \mathrm{fgA}$ & 1.36 & $0.32 \mathrm{f}-\mathrm{hA}$ & $0.30 \mathrm{~g}-\mathrm{iA}$ & 0.31 & $0.26 \mathrm{~d}-\mathrm{fA}$ & $0.25 \mathrm{efA}$ & 0.26 \\
\hline T5 & $2.30 \mathrm{~b}-\mathrm{dA}$ & $2.32 \mathrm{~b}-\mathrm{dA}$ & 2.31 & $1.38 \mathrm{f}-\mathrm{gA}$ & $1.41 \mathrm{fgA}$ & 1.40 & $0.35 \mathrm{e}-\mathrm{gA}$ & $0.35 \mathrm{e}-\mathrm{gA}$ & 0.35 & $0.28 \mathrm{c}-\mathrm{eA}$ & $0.27 \mathrm{~d}-\mathrm{fA}$ & 0.28 \\
\hline T6 & $2.29 \mathrm{cdA}$ & $2.31 \mathrm{~b}-\mathrm{dA}$ & 2.30 & $2.11 \mathrm{dA}$ & $2.00 \mathrm{eB}$ & 2.06 & $0.38 \mathrm{c}-\mathrm{eA}$ & $0.37 \mathrm{~d}-\mathrm{fA}$ & 0.38 & $0.31 \mathrm{~b}-\mathrm{dA}$ & $0.33 \mathrm{bcA}$ & 0.32 \\
\hline $\mathrm{T} 7$ & $2.31 \mathrm{~b}-\mathrm{dA}$ & $2.33 \mathrm{~b}-\mathrm{dA}$ & 2.32 & $2.19 \mathrm{cA}$ & $2.14 \mathrm{cdB}$ & 2.17 & $0.40 \mathrm{c}-\mathrm{eA}$ & $0.41 b c A$ & 0.41 & $0.33 \mathrm{bcA}$ & $0.35 \mathrm{abA}$ & 0.34 \\
\hline $\mathrm{T} 8$ & $2.38 \mathrm{bA}$ & $2.35 \mathrm{bcA}$ & 2.37 & $2.31 \mathrm{abA}$ & $2.29 \mathrm{bA}$ & 2.30 & $0.46 \mathrm{abA}$ & $0.43 \mathrm{bcA}$ & 0.45 & $0.36 \mathrm{abA}$ & $0.36 \mathrm{abA}$ & 0.36 \\
\hline T9 & $2.49 \mathrm{aA}$ & $2.51 \mathrm{aA}$ & 2.50 & $2.36 \mathrm{aA}$ & $2.33 \mathrm{abA}$ & 2.35 & $0.51 \mathrm{aA}$ & $0.49 \mathrm{bA}$ & 0.50 & $0.39 \mathrm{aA}$ & $0.37 \mathrm{aA}$ & 0.38 \\
\hline Mean (G) & 2.20 & 2.18 & ---- & 1.75 & 1.74 & ---- & 0.36 & 0.34 & ---- & 0.29 & 0.29 & ---- \\
\hline \multicolumn{13}{|l|}{ L.S.D. $(0.05)^{2}$} \\
\hline G & \multicolumn{3}{|c|}{ Sig. } & \multicolumn{3}{|c|}{ Sig. } & \multicolumn{3}{|c|}{ Sig. } & \multicolumn{3}{|c|}{ N.S } \\
\hline $\mathrm{T}$ & \multicolumn{3}{|c|}{0.05} & \multicolumn{3}{|c|}{0.03} & \multicolumn{3}{|c|}{0.04} & \multicolumn{3}{|c|}{0.04} \\
\hline $\mathrm{GX} \mathrm{T}$ & \multicolumn{3}{|c|}{0.07} & \multicolumn{3}{|c|}{0.04} & \multicolumn{3}{|c|}{0.06} & \multicolumn{3}{|c|}{0.05} \\
\hline \multicolumn{13}{|c|}{$2^{\text {nd }}$ season } \\
\hline T1 (Control) & $1.42 \mathrm{jB}$ & $1.46 \mathrm{j} \mathrm{A}$ & 1.44 & $1.38 \mathrm{ij} \mathrm{A}$ & $1.31 \mathrm{kB}$ & 1.35 & $0.27 \mathrm{hA}$ & $0.21 \mathrm{iB}$ & 0.24 & 0.23 efA & $0.21 \mathrm{gA}$ & 0.22 \\
\hline $\mathrm{T} 2$ & $2.10 \mathrm{f}-\mathrm{hA}$ & $1.86 \mathrm{iB}$ & 1.98 & $1.47 \mathrm{ghA}$ & $1.30 \mathrm{kB}$ & 1.39 & $0.30 \mathrm{hA}$ & $0.28 \mathrm{hA}$ & 0.29 & $0.26 \mathrm{~d}-\mathrm{fA}$ & $0.22 \mathrm{fB}$ & 0.24 \\
\hline $\mathrm{T} 3$ & $2.01 \mathrm{~g}-\mathrm{iA}$ & $1.96 \mathrm{hiB}$ & 1.99 & $1.41 \mathrm{iA}$ & $1.39 \mathrm{iA}$ & 1.40 & $0.33 \mathrm{f}-\mathrm{hA}$ & $0.29 \mathrm{hA}$ & 0.31 & $0.25 \mathrm{efA}$ & $0.23 \mathrm{efA}$ & 0.24 \\
\hline $\mathrm{T} 4$ & $2.11 \mathrm{f}-\mathrm{hB}$ & $2.15 \mathrm{e}-\mathrm{gA}$ & 2.13 & $1.49 \mathrm{gA}$ & $1.33 \mathrm{jkB}$ & 1.41 & $0.37 \mathrm{e}-\mathrm{gA}$ & $0.30 \mathrm{hB}$ & 0.34 & $0.29 \mathrm{c}-\mathrm{eA}$ & $0.26 \mathrm{~d}-\mathrm{fA}$ & 0.28 \\
\hline T5 & $2.40 \mathrm{bcA}$ & $2.19 \mathrm{~d}-\mathrm{fB}$ & 2.30 & $1.42 \mathrm{hiA}$ & $1.32 \mathrm{kB}$ & 1.37 & $0.40 \mathrm{de} A$ & $0.33 \mathrm{f}-\mathrm{hB}$ & 0.37 & $0.33 \mathrm{bcA}$ & $0.26 \mathrm{~d}-\mathrm{fB}$ & 0.30 \\
\hline T6 & $2.31 \mathrm{c}-\mathrm{eA}$ & $2.18 \mathrm{~d}-\mathrm{fA}$ & 2.25 & $2.05 \mathrm{fA}$ & $2.04 \mathrm{fA}$ & 2.05 & $0.44 \mathrm{cAd}$ & $0.32 \mathrm{f}-\mathrm{hB}$ & 0.38 & $0.33 \mathrm{bcA}$ & $0.33 \mathrm{bcA}$ & 0.33 \\
\hline $\mathrm{T} 7$ & $2.33 \mathrm{cdA}$ & $2.20 \mathrm{~d}-\mathrm{fA}$ & 2.27 & $2.11 \mathrm{eA}$ & $2.15 \mathrm{eA}$ & 2.13 & $0.42 \mathrm{c}-\mathrm{eA}$ & $0.38 \mathrm{efB}$ & 0.40 & $0.36 \mathrm{abA}$ & $0.35 \mathrm{abA}$ & 0.36 \\
\hline $\mathrm{T} 8$ & $2.20 \mathrm{~d}-\mathrm{fA}$ & $2.33 \mathrm{cdA}$ & 2.27 & $2.28 \mathrm{cA}$ & $2.22 \mathrm{~dB}$ & 2.25 & $0.47 \mathrm{bcA}$ & $0.45 \mathrm{cdA}$ & 0.46 & $0.38 \mathrm{bA}$ & $0.35 \mathrm{abB}$ & 0.37 \\
\hline T9 & $2.95 \mathrm{aA}$ & $2.54 \mathrm{bB}$ & 2.75 & $2.49 \mathrm{aA}$ & $2.40 \mathrm{bB}$ & 2.45 & $0.59 \mathrm{aA}$ & $0.55 \mathrm{bA}$ & 0.57 & $0.44 \mathrm{aA}$ & $0.44 \mathrm{aA}$ & 0.44 \\
\hline Mean (G) & 2.20 & 2.10 & ---- & 1.79 & 1.72 & ---- & 0.40 & 0.35 & ---- & 0.32 & 0.29 & ---- \\
\hline \multicolumn{13}{|l|}{ L.S.D. (0.05) * } \\
\hline G & \multicolumn{3}{|c|}{ Sig. } & \multicolumn{3}{|c|}{ Sig. } & \multicolumn{3}{|c|}{ Sig. } & \multicolumn{3}{|c|}{ Sig. } \\
\hline $\mathrm{T}$ & & 0.11 & & & 0.03 & & & 0.05 & & & 0.02 & \\
\hline $\mathrm{GXT}$ & & 0.15 & & & 0.05 & & & 0.06 & & & 0.05 & \\
\hline
\end{tabular}

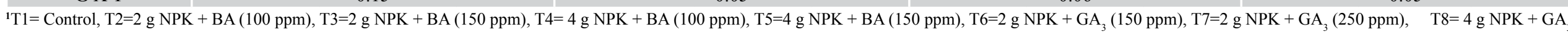

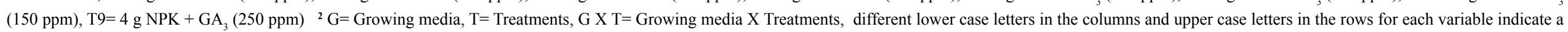
significant difference at $5 \%$ level of significance by LSD test. 


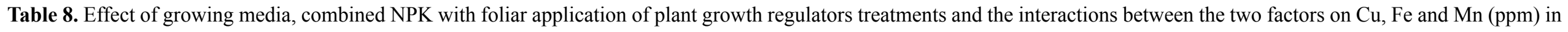
leaves and stems of Dracaena marginata during the 2018 and 2019 seasons.

\begin{tabular}{|c|c|c|c|c|c|c|c|c|c|c|c|c|c|c|c|c|c|c|}
\hline \multirow{4}{*}{$\begin{array}{l}\text { Treatments } \\
(\mathrm{T})^{1}\end{array}$} & \multicolumn{18}{|c|}{$1^{\text {st }}$ season } \\
\hline & \multicolumn{18}{|c|}{ Growing media (G) } \\
\hline & Peat & Peat+ sand & $\begin{array}{l}\text { Mean } \\
(\mathrm{T})\end{array}$ & Peat & Peat+ sand & $\begin{array}{l}\text { Mean } \\
(\mathrm{T})\end{array}$ & Peat & Peat+ sand & $\begin{array}{l}\text { Mean } \\
(\mathrm{T})\end{array}$ & Peat & Peat+ sand & $\begin{array}{l}\text { Mean } \\
(\mathrm{T})\end{array}$ & Peat & Peat + sand & $\begin{array}{l}\text { Mean } \\
(\mathrm{T})\end{array}$ & Peat & $\begin{array}{l}\text { Peat }+ \\
\text { sand }\end{array}$ & $\begin{array}{l}\text { Mean } \\
(\mathrm{T})\end{array}$ \\
\hline & \multicolumn{3}{|c|}{$\mathrm{Cu}(\mathrm{ppm})$ in leaves } & \multicolumn{3}{|c|}{$\mathrm{Cu}(\mathrm{ppm})$ in stems } & \multicolumn{3}{|c|}{$\mathrm{Fe}(\mathrm{ppm})$ in leaves } & \multicolumn{3}{|c|}{$\mathrm{Fe}(\mathrm{ppm})$ in stems } & \multicolumn{3}{|c|}{ Mn (ppm) in leaves } & \multicolumn{3}{|c|}{ Mn (ppm) in stems } \\
\hline T1 (Control) & $4.87 \mathrm{jA}$ & $4.81 \mathrm{jA}$ & 4.84 & $3.22 \mathrm{gA}$ & $3.18 \mathrm{gA}$ & 3.20 & $68.29 \mathrm{rA}$ & $65.23 \mathrm{sB}$ & 66.76 & $50.13 q B$ & $52.10 \mathrm{pA}$ & 51.12 & $53.28 \mathrm{oA}$ & $50.89 \mathrm{pB}$ & 52.09 & $48.99 \mathrm{iB}$ & $51.11 \mathrm{hA}$ & 50.05 \\
\hline $\mathrm{T} 2$ & $5.75 \mathrm{~h}-\mathrm{jA}$ & $5.25 \operatorname{igA}$ & 5.50 & $3.55 \mathrm{gA}$ & $3.49 \mathrm{gA}$ & 3.52 & $86.860 \mathrm{~A}$ & $81.74 \mathrm{pB}$ & 84.30 & $52.21 \mathrm{oB}$ & $55.27 \mathrm{nA}$ & 53.74 & $66.91 \mathrm{~mA}$ & $63.79 \mathrm{nB}$ & 65.35 & $59.22 \mathrm{fA}$ & $55.27 \mathrm{gB}$ & 57.25 \\
\hline $\mathrm{T} 3$ & 5.89hiA & $6.39 \mathrm{ghA}$ & 6.14 & $3.78 \mathrm{gA}$ & $4.28 \mathrm{fA}$ & 4.03 & $98.71 \mathrm{~mA}$ & $95.37 \mathrm{nB}$ & 97.04 & $60.121 \mathrm{~A}$ & $57.19 \mathrm{mB}$ & 58.66 & 69.111A & $66.48 \mathrm{mB}$ & 67.80 & $56.13 \mathrm{gB}$ & $59.10 \mathrm{fA}$ & 57.62 \\
\hline $\mathrm{T} 4$ & $7.33 \mathrm{gA}$ & $7.21 \mathrm{gA}$ & 7.27 & $4.28 \mathrm{fB}$ & $5.63 \mathrm{eA}$ & 4.96 & $114.521 \mathrm{~B}$ & $117.11 \mathrm{kA}$ & 115.82 & $59.95 \mathrm{kB}$ & $62.05 \mathrm{jA}$ & 61.00 & $80.36 \mathrm{hB}$ & $87.96 \mathrm{gA}$ & 84.16 & $64.16 \mathrm{eA}$ & $65.19 \mathrm{eA}$ & 64.68 \\
\hline T5 & $8.97 \mathrm{fA}$ & $9.43 \mathrm{fA}$ & 9.20 & $6.13 \mathrm{dA}$ & $6.09 \mathrm{dA}$ & 6.11 & $122.71 \mathrm{jA}$ & $120.13 \mathrm{iB}$ & 121.42 & $63.22 \mathrm{iB}$ & $68.43 \mathrm{hA}$ & 65.83 & $88.12 \mathrm{fB}$ & $90.32 \mathrm{dA}$ & 89.22 & $70.12 \mathrm{dA}$ & $71.22 \mathrm{dA}$ & 70.67 \\
\hline T6 & $10.95 \mathrm{eB}$ & $13.91 \mathrm{cA}$ & 12.43 & $7.59 \mathrm{cdB}$ & $9.47 \mathrm{bA}$ & 8.53 & $133.63 \mathrm{hB}$ & $136.29 \mathrm{gA}$ & 134.96 & $80.34 \mathrm{gB}$ & $84.71 \mathrm{fA}$ & 82.53 & $71.42 \mathrm{kB}$ & $78.42 \mathrm{iA}$ & 74.92 & $72.11 \mathrm{dA}$ & 71.61dA & 71.86 \\
\hline $\mathrm{T} 7$ & $10.61 \mathrm{eB}$ & $12.81 \mathrm{dA}$ & 11.71 & $8.02 \mathrm{cdA}$ & $8.75 \mathrm{cdA}$ & 8.39 & $151.82 \mathrm{fB}$ & $155.32 \mathrm{eA}$ & 153.57 & $85.02 \mathrm{eA}$ & $84.69 \mathrm{eA}$ & 84.86 & 75.33jB & $82.19 \mathrm{gA}$ & 78.76 & $70.49 \mathrm{dA}$ & $69.94 \mathrm{eA}$ & 70.22 \\
\hline $\mathrm{T} 8$ & $14.29 \mathrm{cA}$ & $14.10 \mathrm{cA}$ & 14.20 & $8.15 \mathrm{bcA}$ & $9.95 \mathrm{bA}$ & 9.05 & $167.33 \mathrm{~dB}$ & $170.11 \mathrm{cA}$ & 168.72 & $93.10 \mathrm{~dB}$ & $95.00 \mathrm{cA}$ & 94.05 & $89.96 \mathrm{eB}$ & $97.82 \mathrm{bA}$ & 93.89 & $75.52 \mathrm{cA}$ & $74.33 \mathrm{cB}$ & 74.93 \\
\hline T9 & $20.45 \mathrm{aA}$ & $18.14 \mathrm{bB}$ & 19.30 & $11.11 \mathrm{aA}$ & $10.01 \mathrm{abA}$ & 10.56 & $180.54 \mathrm{bB}$ & $189.04 \mathrm{aA}$ & 184.79 & $96.37 \mathrm{bB}$ & $98.35 \mathrm{aA}$ & 97.36 & $94.85 \mathrm{cB}$ & $100.1 \mathrm{aA}$ & 97.49 & $79.13 \mathrm{bB}$ & $81.07 \mathrm{aA}$ & 80.10 \\
\hline Mean (G) & 9.90 & 10.23 & ---- & 6.20 & 6.76 & ---- & 124.93 & 125.59 & ---- & 71.16 & 73.09 & ---- & 76.59 & 79.78 & ---- & 66.21 & 66.54 & ---- \\
\hline \multicolumn{19}{|l|}{$\begin{array}{l}\text { L.S.D. } \\
(0.05)^{2}\end{array}$} \\
\hline G & \multicolumn{3}{|c|}{ Sig. } & \multicolumn{3}{|c|}{ Sig. } & \multicolumn{3}{|c|}{ Sig. } & \multicolumn{3}{|c|}{ Sig. } & \multicolumn{3}{|c|}{ Sig. } & \multicolumn{3}{|c|}{ Sig. } \\
\hline $\mathrm{T}$ & \multicolumn{3}{|c|}{0.64} & \multicolumn{3}{|c|}{0.70} & \multicolumn{3}{|c|}{0.58} & \multicolumn{3}{|c|}{0.48} & \multicolumn{3}{|c|}{0.71} & \multicolumn{3}{|c|}{0.87} \\
\hline GX T & \multicolumn{3}{|c|}{0.91} & & 0.99 & & & 0.82 & & & 0.68 & & & 1.01 & & & 1.23 & \\
\hline & & & & & & & & $2^{\text {nd }} s$ & ason & & & & & & & & & \\
\hline T1 (Control) & $4.46 \mathrm{hA}$ & $4.44 \mathrm{hA}$ & 4.45 & $2.89 \mathrm{gA}$ & $2.73 \mathrm{gA}$ & 2.81 & $74.11 \mathrm{pA}$ & $73.29 \mathrm{qA}$ & 73.70 & $61.67 \mathrm{jA}$ & $49.92 \mathrm{oB}$ & 55.80 & 57.741A & $48.47 \mathrm{mB}$ & 53.11 & $44.22 \mathrm{kA}$ & $43.681 \mathrm{~A}$ & 43.95 \\
\hline $\mathrm{T} 2$ & $5.42 \mathrm{gA}$ & $5.53 \mathrm{gA}$ & 5.48 & $3.15 \mathrm{fA}$ & $3.45 \mathrm{fA}$ & 3.30 & $86.00 \mathrm{oB}$ & $100.91 \mathrm{~mA}$ & 93.46 & 58.031A & $51.19 \mathrm{nB}$ & 54.61 & $68.98 \mathrm{iA}$ & $66.39 \mathrm{kB}$ & 67.69 & $54.82 \mathrm{jA}$ & $54.19 \mathrm{jA}$ & 54.51 \\
\hline $\mathrm{T} 3$ & $5.54 \mathrm{gB}$ & $6.45 \mathrm{fA}$ & 6.00 & $3.65 \mathrm{fA}$ & $3.77 \mathrm{fA}$ & 3.71 & $102.821 \mathrm{~A}$ & $97.32 \mathrm{nB}$ & 100.07 & $65.53 \mathrm{hA}$ & $52.61 \mathrm{lmB}$ & 59.07 & $75.95 \mathrm{hA}$ & $67.16 \mathrm{jB}$ & 71.56 & $54.38 \mathrm{jB}$ & $58.51 \mathrm{hA}$ & 56.45 \\
\hline $\mathrm{T} 4$ & $6.54 \mathrm{fA}$ & $6.61 \mathrm{fA}$ & 6.58 & $3.94 \mathrm{fA}$ & $4.55 \mathrm{eA}$ & 4.25 & $137.98 \mathrm{iA}$ & $120.12 \mathrm{kB}$ & 129.05 & $64.15 \mathrm{iA}$ & $59.36 \mathrm{kB}$ & 61.76 & $82.99 \mathrm{fB}$ & $87.40 \mathrm{dA}$ & 85.20 & $56.28 \mathrm{iB}$ & $70.10 \mathrm{dA}$ & 63.19 \\
\hline T5 & $9.63 \mathrm{dA}$ & $8.98 \mathrm{eA}$ & 9.31 & $6.62 \mathrm{dA}$ & $4.39 \mathrm{eB}$ & 5.51 & $133.38 \mathrm{jB}$ & $138.08 \mathrm{hA}$ & 135.73 & $65.52 \mathrm{hB}$ & 73.64fA & 69.58 & $91.79 \mathrm{cA}$ & $85.21 \mathrm{eB}$ & 88.50 & $68.08 \mathrm{fA}$ & $69.93 \mathrm{eB}$ & 69.01 \\
\hline T6 & $9.36 \mathrm{dA}$ & $12.88 \mathrm{bB}$ & 11.12 & $6.29 \mathrm{~d}$ & $9.21 \mathrm{abA}$ & 7.75 & $139.73 \mathrm{fA}$ & $131.55 \mathrm{gB}$ & 135.64 & $66.68 \mathrm{gB}$ & $73.53 \mathrm{fA}$ & 70.11 & $75.18 \mathrm{hA}$ & $73.98 \mathrm{~A}$ & 74.58 & $74.34 \mathrm{cA}$ & $64.21 \mathrm{gB}$ & 69.28 \\
\hline $\mathrm{T} 7$ & $11.61 \mathrm{cA}$ & $12.20 \mathrm{bA}$ & 11.91 & $7.54 \mathrm{cB}$ & $8.61 \mathrm{bA}$ & 8.08 & $153.35 \mathrm{eA}$ & $153.78 \mathrm{eA}$ & 153.57 & $83.32 \mathrm{~dB}$ & $89.99 \mathrm{bA}$ & 86.66 & $76.09 \mathrm{gA}$ & $75.54 \mathrm{hA}$ & 75.82 & $73.43 \mathrm{cA}$ & $68.77 \mathrm{fB}$ & 71.10 \\
\hline $\mathrm{T} 8$ & $13.74 \mathrm{bA}$ & $11.75 \mathrm{cB}$ & 12.75 & $6.35 \mathrm{~dB}$ & $8.97 \mathrm{bA}$ & 7.66 & $165.67 \mathrm{~dB}$ & $193.13 \mathrm{bA}$ & 179.40 & $77.27 \mathrm{eB}$ & $89.15 \mathrm{cA}$ & 83.21 & $93.87 \mathrm{cB}$ & $115.2 \mathrm{bA}$ & 104.53 & $64.93 \mathrm{gB}$ & $77.43 \mathrm{bA}$ & 71.18 \\
\hline T9 & $15.29 \mathrm{aA}$ & $15.32 \mathrm{aA}$ & 15.31 & $9.44 \mathrm{aA}$ & $8.31 \mathrm{bB}$ & 8.88 & $186.43 \mathrm{cB}$ & $207.74 \mathrm{aA}$ & 197.09 & $82.28 \mathrm{db}$ & $95.47 \mathrm{aA}$ & 88.88 & $92.79 \mathrm{cB}$ & $117.8 \mathrm{aA}$ & 105.30 & $73.58 \mathrm{cB}$ & $83.83 \mathrm{aA}$ & 78.71 \\
\hline Mean (G) & 9.07 & 9.35 & ---- & 5.54 & 6.00 & --- & 131.05 & 135.10 & ---- & 69.38 & 70.54 & ---- & 79.49 & 81.90 & ---- & 62.67 & 65.63 & ---- \\
\hline $\begin{array}{l}\text { L.S.D. } \\
(0.05)^{*}\end{array}$ & & & & & & & & & & & & & & & & & & \\
\hline G & & Sig. & & & Sig. & & & Sig. & & & Sig. & & & Sig. & & & Sig. & \\
\hline $\mathrm{T}$ & & 0.56 & & & 0.61 & & & 0.71 & & & 0.36 & & & 0.93 & & & 0.97 & \\
\hline GX T & & 0.79 & & & 0.86 & & & 1.00 & & & 0.51 & & & 1.31 & & & 1.37 & \\
\hline
\end{tabular}

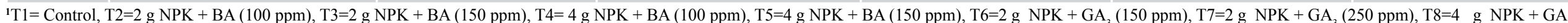

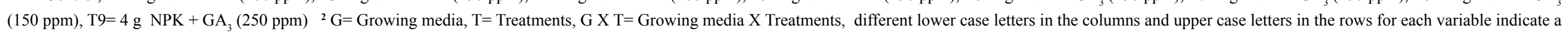
significant difference at $5 \%$ level of significance by LSD test. 


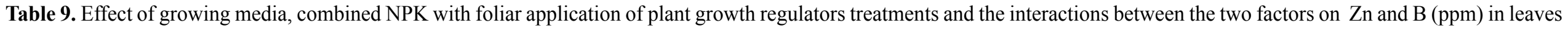
and stems of Dracaena marginata during the 2018 and 2019 seasons.

\begin{tabular}{|c|c|c|c|c|c|c|c|c|c|c|c|c|}
\hline \multirow{4}{*}{ Treatments $(T)^{1}$} & \multicolumn{12}{|c|}{$1^{\text {st }}$ season } \\
\hline & \multicolumn{12}{|c|}{ Growing media (G) } \\
\hline & Peat & Peat + sand & Mean $(\mathrm{T})$ & Peat & Peat + sand & Mean $(T)$ & Peat & Peat + sand & Mean $(\mathrm{T})$ & Peat & Peat + sand & Mean $(\mathrm{T})$ \\
\hline & \multicolumn{3}{|c|}{$\mathrm{Zn}(\mathbf{p p m})$ in leaves } & \multicolumn{3}{|c|}{$\mathrm{Zn}(\mathbf{p p m})$ in stems } & \multicolumn{3}{|c|}{ B (ppm) in leaves } & \multicolumn{3}{|c|}{ B (ppm) in stems } \\
\hline T1 (Control) & $41.27 \mathrm{qB}$ & $43.63 \mathrm{nA}$ & 42.45 & $20.39 \mathrm{sB}$ & $23.14 \mathrm{qA}$ & 21.77 & $19.05 \mathrm{sB}$ & $20.27 \mathrm{qA}$ & 19.66 & $18.87 \mathrm{pA}$ & $18.02 \mathrm{qB}$ & 18.45 \\
\hline $\mathrm{T} 2$ & $42.52 \mathrm{pB}$ & $49.12 \mathrm{~mA}$ & 45.82 & $35.12 \mathrm{nA}$ & $28.34 \mathrm{pB}$ & 31.73 & $22.91 \mathrm{pB}$ & $24.62 \mathrm{oA}$ & 23.77 & $21.36 \mathrm{nA}$ & $20.16 \mathrm{oB}$ & 20.76 \\
\hline $\mathrm{T} 3$ & $44.93 \mathrm{nB}$ & 53.13jA & 49.03 & $37.33 \mathrm{~mA}$ & $33.27 \mathrm{oB}$ & 35.30 & $25.39 \mathrm{nB}$ & $28.13 \mathrm{~mA}$ & 26.76 & $24.101 \mathrm{~A}$ & $22.10 \mathrm{mB}$ & 23.10 \\
\hline $\mathrm{T} 4$ & $48.7 \mathrm{lB}$ & $58.37 \mathrm{kA}$ & 53.57 & $40.11 \mathrm{kA}$ & $39.421 \mathrm{~B}$ & 39.77 & $29.471 \mathrm{~B}$ & $31.41 \mathrm{kA}$ & 30.44 & $26.90 \mathrm{jA}$ & $24.41 \mathrm{kB}$ & 25.655 \\
\hline T5 & $53.11 \mathrm{jB}$ & $61.19 \mathrm{iA}$ & 57.15 & $45.81 \mathrm{iA}$ & $43.88 \mathrm{jB}$ & 44.85 & $32.49 \mathrm{jB}$ & $35.61 \mathrm{iA}$ & 34.05 & $30.01 \mathrm{hA}$ & $27.20 \mathrm{iB}$ & 28.605 \\
\hline T6 & $61.53 \mathrm{hB}$ & $67.55 \mathrm{gA}$ & 64.54 & $49.13 \mathrm{hB}$ & $52.43 \mathrm{gA}$ & 50.78 & $37.86 \mathrm{hB}$ & $39.52 \mathrm{gA}$ & 38.69 & $33.17 \mathrm{fA}$ & $29.07 \mathrm{~B}$ & 31.12 \\
\hline $\mathrm{T} 7$ & $69.18 \mathrm{fB}$ & $73.39 \mathrm{eA}$ & 71.29 & $54.26 \mathrm{fB}$ & $56.36 \mathrm{eA}$ & 55.31 & $41.93 \mathrm{fB}$ & $44.21 \mathrm{eA}$ & 43.07 & $36.08 \mathrm{dA}$ & $31.14 \mathrm{gB}$ & 33.61 \\
\hline $\mathrm{T} 8$ & $78.21 \mathrm{~dB}$ & $80.63 \mathrm{cA}$ & 79.42 & $59.20 \mathrm{~dB}$ & $60.32 \mathrm{cA}$ & 59.76 & $44.13 \mathrm{~dB}$ & $47.19 \mathrm{cA}$ & 45.66 & $39.03 \mathrm{bA}$ & $34.27 \mathrm{eB}$ & 36.65 \\
\hline T9 & $89.60 \mathrm{bB}$ & $91.47 \mathrm{aA}$ & 90.54 & $66.10 \mathrm{aA}$ & $63.29 \mathrm{bB}$ & 64.70 & $48.29 \mathrm{bB}$ & $49.11 \mathrm{aA}$ & 48.70 & $41.23 \mathrm{aA}$ & $38.10 \mathrm{cB}$ & 39.67 \\
\hline Mean (G) & 58.79 & 64.28 & ---- & 45.27 & 44.49 & ---- & 33.50 & 35.56 & --- & 30.08 & 27.16 & --- \\
\hline \multicolumn{13}{|l|}{ L.S.D. $(0.05)^{2}$} \\
\hline G & \multicolumn{3}{|c|}{ Sig. } & \multicolumn{3}{|c|}{ Sig. } & \multicolumn{3}{|c|}{ Sig. } & \multicolumn{3}{|c|}{ Sig. } \\
\hline $\mathrm{T}$ & \multicolumn{3}{|c|}{0.83} & \multicolumn{3}{|c|}{0.38} & \multicolumn{3}{|c|}{0.04} & \multicolumn{3}{|c|}{0.14} \\
\hline GX T & \multicolumn{3}{|c|}{1.17} & \multicolumn{3}{|c|}{0.54} & \multicolumn{3}{|c|}{0.05} & \multicolumn{3}{|c|}{0.20} \\
\hline \multicolumn{13}{|c|}{$2^{\text {nd }}$ season } \\
\hline T1 (Control) & $40.20 \mathrm{pB}$ & $43.93 \mathrm{oA}$ & 42.07 & $24.17 \mathrm{oB}$ & $25.88 \mathrm{nA}$ & 25.03 & $19.86 \mathrm{qB}$ & $25.560 \mathrm{~A}$ & 22.71 & $23.3 \mathrm{oA}$ & $20.45 \mathrm{qB}$ & 21.88 \\
\hline $\mathrm{T} 2$ & $50.85 \mathrm{nB}$ & $56.02 \mathrm{kA}$ & 53.44 & $45.03 \mathrm{kA}$ & $36.81 \mathrm{mB}$ & 40.92 & $24.90 \mathrm{pB}$ & $27.05 \mathrm{nA}$ & 25.98 & $24.05 \mathrm{nA}$ & $21.45 \mathrm{pB}$ & 22.75 \\
\hline $\mathrm{T} 3$ & $58.61 \mathrm{iB}$ & $57.26 \mathrm{jA}$ & 57.94 & $49.12 \mathrm{iA}$ & $41.07 \mathrm{lB}$ & 45.10 & $27.90 \mathrm{mB}$ & $32.33 \mathrm{jA}$ & 30.12 & $25.91 \mathrm{mB}$ & $26.111 \mathrm{~A}$ & 26.01 \\
\hline $\mathrm{T} 4$ & $58.93 \mathrm{fB}$ & $59.81 \mathrm{gA}$ & 59.37 & $54.95 \mathrm{gA}$ & $44.80 \mathrm{kB}$ & 49.88 & $34.27 \mathrm{iA}$ & $30.791 \mathrm{~B}$ & 32.53 & $31.65 \mathrm{kB}$ & $32.12 \mathrm{jA}$ & 31.89 \\
\hline T5 & $54.611 \mathrm{~A}$ & $52.43 \mathrm{mB}$ & 53.52 & $53.08 \mathrm{hA}$ & $49.68 \mathrm{iB}$ & 51.38 & $30.101 \mathrm{~B}$ & $35.97 \mathrm{hA}$ & 33.04 & $34.10 \mathrm{hB}$ & $36.27 \mathrm{eA}$ & 35.19 \\
\hline T6 & $66.30 \mathrm{cA}$ & $59.30 \mathrm{hB}$ & 62.80 & 47.30jB & $57.62 \mathrm{eA}$ & 52.46 & $35.49 \mathrm{gB}$ & $41.17 f \mathrm{~A}$ & 38.33 & $34.57 \mathrm{gA}$ & 33.93iB & 34.25 \\
\hline $\mathrm{T} 7$ & $63.74 \mathrm{dA}$ & $62.16 \mathrm{eB}$ & 62.95 & $56.17 \mathrm{fB}$ & $59.96 \mathrm{dA}$ & 58.07 & $42.35 \mathrm{fB}$ & $46.05 \mathrm{dA}$ & 44.20 & $35.10 \mathrm{fB}$ & $36.48 \mathrm{dA}$ & 35.79 \\
\hline $\mathrm{T} 8$ & $56.88 \mathrm{kB}$ & $69.86 \mathrm{bA}$ & 63.37 & $61.48 \mathrm{cB}$ & $62.67 \mathrm{bA}$ & 62.08 & $44.58 \mathrm{eB}$ & $47.67 \mathrm{cA}$ & 46.13 & $41.97 \mathrm{~dB}$ & $44.32 \mathrm{bA}$ & 43.15 \\
\hline T9 & $56.24 \mathrm{kB}$ & $75.69 \mathrm{aA}$ & 65.97 & $61.97 \mathrm{cB}$ & $78.41 \mathrm{aA}$ & 70.19 & $51.37 \mathrm{bB}$ & $53.63 \mathrm{aA}$ & 52.50 & $43.55 \mathrm{cB}$ & $46.41 \mathrm{aA}$ & 44.98 \\
\hline Mean (G) & 56.26 & 59.61 & ---- & 50.36 & 50.77 & ---- & 34.54 & 37.80 & ---- & 32.69 & 33.06 & ---- \\
\hline \multicolumn{13}{|l|}{ L.S.D. $(0.05)^{*}$} \\
\hline G & \multicolumn{3}{|c|}{ Sig. } & \multicolumn{3}{|c|}{ Sig. } & \multicolumn{3}{|c|}{ Sig. } & \multicolumn{3}{|c|}{ Sig. } \\
\hline $\mathrm{T}$ & & 0.86 & & & 0.55 & & & 0.15 & & & 0.11 & \\
\hline G X T & & 1.21 & & & 0.78 & & & 0.11 & & & 0.16 & \\
\hline
\end{tabular}

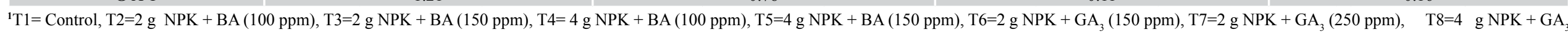

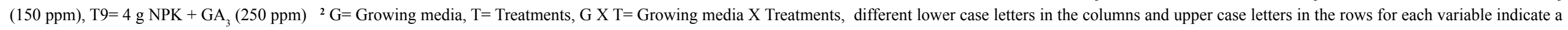
significant difference at $5 \%$ level of significance by LSD test. 
In this respect previous finding (Abdul-Hafeez et al., 2015) indicated that peat-grown plants were inferior to those grown in clay or rice straw in $\mathrm{Fe}, \mathrm{Cu}$ and $\mathrm{Mn}$ contents in leaves of Gardenia jasminoides.

The data in in Tables (8 and 9) also cleared that the uptake and accumulation of $\mathrm{Cu}, \mathrm{Fe}, \mathrm{Mn}, \mathrm{Zn}$ and $\mathrm{B}$ contents in the leaves and stems were markedly affected by the treatments of combined NPK with both BA and $\mathrm{GA}_{3}$ as compared to the control plants. In both seasons, in most cases plants treated with any of the combined treatments had significantly higher values than the values obtained from untreated control plants. Also the data indicated that, $\mathrm{GA}_{3}$ was more effective than BA when combined with NPK at the same rates.

Concerning the interaction effects, the data in Tables (8 and 9) revealed that plants received any treatments of combined NPK with either BA or GA had significantly higher values of micro elements than those obtained from control plants. Overall, in both seasons the lowest values were obtained from plants grown in medium containing peat-moss only or peat-moss + sand and received no treatments. While, the highest values were recorded with plants grown in medium of peat- moss + sand and received combined treatments of $4 \mathrm{~g} \mathrm{NPK}+\mathrm{GA}_{3}$ at $250 \mathrm{ppm}$.

\section{Leaf anatomy}

As shown in Fig.1 (a, b, and c) the leaves of Dracaena marginata are compound and composed of two little thicker upper and lower epidermis cells. They are covered with thick layer of cuticle particularly existing in the upper epidermis, meanwhile stomata were concentrated on the lower epidermis.

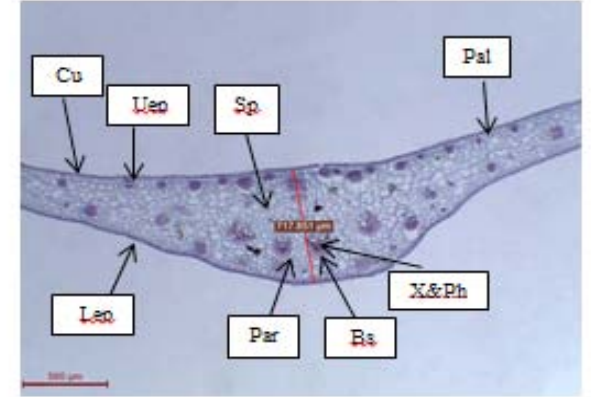

a. (control)

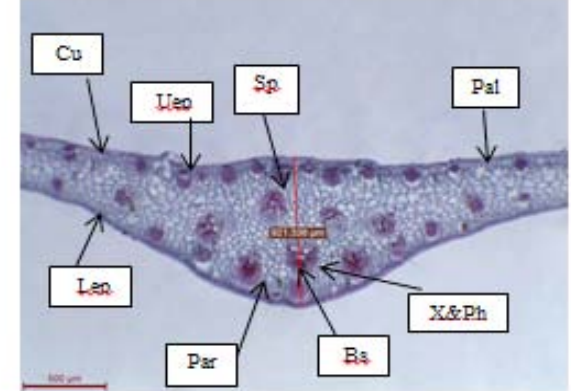

b. (NPK with BA)

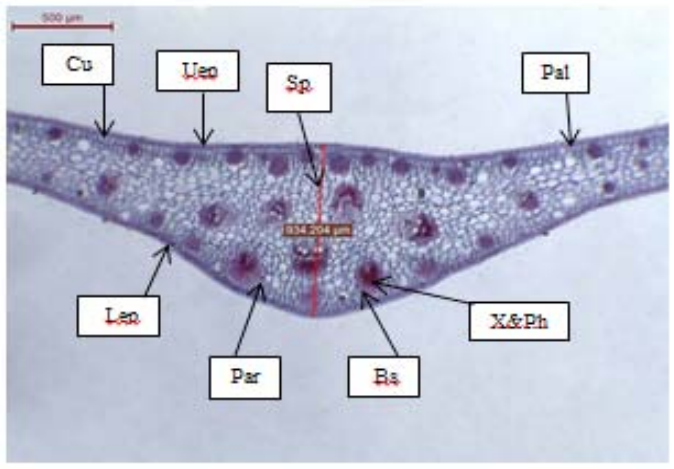

c. (NPK with GA3)

Figure 1. $\mathrm{Cu}$ : cuticle, $\underline{\text { Uep: }}$ upper epidermis, $\underline{\mathrm{Sp}}$ : spongy parenchyma, Pal: palisade tissue, Lep: lower epidermis,

$\underline{\text { Par: }}$ parenchyma, $\underline{B s}$ : bundle sheath, $\underline{X \& P h}$ : xylem and phloem.

Cross section in the leaves revealed that, the epidermis tissue cells (both upper and lower) of the control (Fig. a) were consisting of similar shape and size barrel, compactly arranged tiny cells and then become thicker and larger than control as shown in (Fig. b) as a result of the combined NPK with BA treatments. The application of NPK combined with $\mathrm{GA}_{3}$ treatments resulted in the maximum size of the epidermis cells (Fig. c). Regarding the mesophyll tissue that consists of palisade and spongy parenchyma cells, in which the palisade lies just inner to the upper epidermis, and composed of elongated cells organized in two layers. The palisade cells region was compact and filled with chloroplasts which arranged along their radial walls, whereas the parenchyma cells tissue were present above and below the large vascular bundles. The spongy parenchyma cells region are loosely arranged, filled with chloroplasts and present below the palisade and extends up to the lower epidermis. It was remarkable that, the thickness of mesophyll tissue, which is specialized in photosynthetic and contains chloroplasts in palisade cells were visible and 
clear. The size of the spongy parenchyma tissue, air spaces of that treated with combined NPK with GA became the largest as compared to both of combined NPK with BA treatments and control. Furthermore, vascular bundles of combined NPK with $\mathrm{GA}_{3}$ treatment were larger and greater as shown in (Fig. c) as compared with the other treatments including control. These results are representing evidence of chlorophyll concentration, which was higher for the combined NPK with $\mathrm{GA}_{3}$ treatment and that reflected the increased total carbohydrates percentage with the same previous treatment compared to control. This might be attributed to the increase in the chlorophylls synthesis and photosynthesis rate. The previous data are in conformity with the results obtained by (Ullah et al., 2017).

\section{Conclusions}

On the basis of earlier stated data, for the best vegetative growth and economic production of Dracaena marginata 'Bicolor', the plants advised to grow in a medium of peatmoss and supplied monthly with NPK fertilizer (20:20:20) at the rate of $2 \mathrm{~g} \mathrm{plant}^{-1}$ along with foliar sprayed of $\mathrm{GA}_{3}$ at the concentration $250 \mathrm{ppm}$.

\section{Author Contribution}

HAA: Responsible for implantation of the experiment according to established treatments, data collection, and data evaluation, arrangement of experimental data, statistical analysis, manuscript writing and corrections. ABE: Implantation of the experiment according to established treatments, data collection, statistical analysis, manuscript writing and corrections. MMA: Assistance in data collection, chemical analysis, statistical analysis, assistance in the preparation of manuscript.

\section{Acknowledgments}

The authors would like to give full thanks to Cairo University, Faculty of Agriculture Research Park (FA-CURP,) and Plant physiology lab as well for giving all facilities and apparatuses to finish present research.

\section{References}

ABD EL GAYED, M.E.; ATTIA, E.A. Impact of growing media and compound fertilizer rates on growth andflowering of cocks comb (Celosia argentea) plants. Journal of Plant Production, v.9, n.11, p.895-900, 2018. DOI: 10.21608/ jpp.2018.36599

ABDUL-HAFEEZ, E.Y; IBRAHIM, O.H.M.; ELKELTAWI, N.E. Reuse of wastewater from phosphate fertilizer factories can combat soil alkalinity and improve quality of potted gardenia (Gardenia jasminoides Ellis). Journal of Biodiversity and Environmental Sciences, v.6, n.3, p.423-433, 2015.
ABOU DAHAB, T.A.M.; ASHOUR, H.A.; EL-DEEB, E.E.A.; SABER, M.M.H. Response of Chamaedorea elegans, Mart. plants grown under different light intensity levels to chemical and organic fertilization treatments. Journal of Horticultural Science \& Ornamental Plants, v.9, n.2, p.72-85, 2017. DOI: 10.5829/idosi.jhsop.2017.72.85

AIKEN, S.O.; DARBYSHIRE, S.J.; LEFKOVITCH, L. P. The taxonomic value of using epidermal characteristics in Canadian rough fescue complex (Festuca altaica, F. campestris, $F$. halii, F. scabrella). Canadian Journal of Botany, v.62, n.9, p.1864-1870, 1984.

ASKARI-KHORASGANI，O.; MORTAZAEINEZHAD, O.F. Improving quality and quantity indices of Rosa 'Yellow Finesse' using methyl jasmonate and benzyl adenine. Journal of Central European Agriculture, v.17, n.2, p. 369-378, 2016. DOI: 10.5513/JCEA01/17.2.1717

BADRAN, F.S.; ABDOU, M.A.; EL-SAYED, A.A; ELSAYED B.A.; GOHAR, A.A. Effect of growing media and fertilization treatments on growth and flowering of gardenia jasminoides plants. Scientific Journal of Flowers \& Ornamental Plants, v.4, n.1, p.131-141, 2017. DOI: $10.21608 /$ sjfop. 2017.5400

BARMAN, D.; NAIK, S.K. Effect of substrate, nutrition and growth regulator on productivity and mineral composition of leaf and pseudobulb of Cymbidium hybrid 'Baltic glacier mint ice'. Journal of Plant Nutrition, v.40, n.6, p.784-794. 2017. DOI: https://doi.org/10.1080/019041 67.2016 .1201496

CHAPMAN, H.D.; PRATT, P.F. Methods of analysis for soil, plants and water. Division of Agriculture Science. Riverside: University of California., 1961. 309p.

DI BENEDETTO, A.; GALMARINI, C. Exogenous cytokinin promotes Epipremnum aureum 1. growth through enhanced dry weight assimilation rather than through changes in partitioning. American Journal of Experimental Agriculture, v.5, n.5, p.419-434, 2015. DOI: https://doi.org/10.9734/AJEA/2015/13398

DUBOIS, M.; SMITH, F.; GILLES, K.A.; HAMILTON J.K.; REBERS, P.A. Colorimetric method for determination of sugar and related substances. Analytical Chemistry, v.28, n.3, p.350-356, 1956.

ELLIS, R.P. A Procedure for standardizing comparative leaf anatomy in the Poaceae, I. The leaf-blade as viewed in transverse section. Bothalia, v.12, n.1, p.65-109, 1976.

EL-NAGGAR, A.H.; AHMAD, Y.A.A. The effect of light intensity and NPK fertilization on growth of Yucca rupicola, L. Journal of Advanced Studies in Agricultural, Biological and Environmental Sciences, v.3, n.1, p.32-41, 2016. 
EL-QUASNI, F. E. M.; MAZHAR, A.M.; SAKR, S.S.; ELKHATEEB, M.A.; ABD EL- MAGIED, H.M. Effect of some growing media on growth and chemical constituents of magnolia seedlings. (Magnolia grandiflora L.). MiddleEast Journal of Scientific Research, v.3, n.4, p.869-875, 2014.

EL-SAYED, B.A.; NOOR EL-DEEN, T.M; ABDELGALEIL, L.M.; EL-ASHWAH, M.A. Effect of NPK and gibberellic acid on growth and quality of Cycas revoluta "thunb". Scientific Journal of Flowers \& Ornamental Plants, v.3, n.1, p.87-94, 2016. DOI: 10.21608/ sjfop. 2016.5126

EL-SAYED, S.G.; ISMAIL, S.M. Influence of bio and chemical fertilization on Croton production. Assiut Journal of Agricultural Sciences, v.48, n.3, p. 112-122, 2017.

ESTEFAN, G.; SOMMER, R.; RYAN, J. Methods of Soil, Plant, and Water Analysis: A manual for the West Asia and North Africa region, 3ed. Beirut: ICARDA, 2013. $244 p$.

FASCELLA, G. Growing substrates alternative to peat for ornamental plants. In: ASADUZZAMAN, Md. Soilless Culture - Use of Substrates for the Production of Quality Horticultural Crops. Rijeka: InTech, 2015. p.47-67.

FUADI, M.; MOHAMED, M.T.M.; SALLEH, N.S.; ANWAR, M.P.; AWANG, Y.; FAUZI, R.M. Effect of different concentrations of benzyladenine and frequency of watering on growth and quality of Dracaena sanderiana and Codiaeum variegatum. Journal of Environmental Biology, v.35, n.6, p.1047-1052, 2014.

GABREL, F.; KHATTAB, M.; EL NAGGAR, A. Effect of benzyl adenine and gibberellic acid on the vegetative growth and flowering of Chrysanthemum plant. Alexandria Journal of Agricultural Sciences, v.63, n.1, p.29-40, 2018. DOI: 10.21608/alexja.2018.30051

GAD, M.M.; ABDUL-HAFEEZ, E.Y.; IBRAHIM, O.H.M. Foliar application of salicylic acid and gibberellic acid enhances growth and flowering of Ixora coccinea 1. plants. Journal of Plant Production, v.7, n.1, p.85-91, 2016.

GHATAS, Y.A.A. Effect of $\mathrm{GA}_{3}$ and chemical fertilization treatments on growth, flowering, corm production and chemical composition of Gladiolus grandiflorus plant. Journal of Plant Production, v.7, n.6, p.627-636, 2016. DOI: $10.21608 /$ jpp.2016.45544

GUPTA, U.C. Determination of boron, molybdenum, and selenium in Plant Tissue. In: KALRA, Y.P. Handbook of reference methods for plant analysis. Boca Raton: CRC Press, Taylor \& Francis Group, 1998. p.171-174.
HANANFY, M.S.; SHANAN N.T.; KORIUM, D.A. Evaluate the effects of gibberellic acid and some natural extracts on the morphological features and anatomical structure of Ficus benjamina L. plants. Plant Archives, v.19, n.1, p. 251-259, 2019.

HENSCHKE, M.; CZUCHAJ, P.K.; SZCZEPANIAK, S.J. The effect of benzyladenine and gibberellic acid on the growth and flowering of Helleborus orientalis Lam. Bulgarian Journal of Agricultural Science, v.21, n.6, p.1198-1203, 2015.

HORNECK, D.A.; HANSON D. Determination of potassium and sodium by Flame spectrophotometry. In: KALRA, Y.P. Handbook of reference methods for plant analysis. Boca Raton: CRC Press, Taylor \& Francis Group, 1998. p.153-155.

HORNECK, D.A.; MILLER, R.O. Determination of total nitrogen in plant tissue. In: KALRA, Y.P. Handbook of reference methods for plant analysis. Boca Raton: CRC Press, Taylor \& Francis Group, 1998. p.75-83.

HUANG, C.T.; LIN C.L.; HSIEH, C.F. Gibberellininduced flowering in sexually defective Remusatia vivipara (Araceae). Taiwania, v.60, n.1, p.1 -7, 2015. DOI: 10.6165/tai.2015.60.1.1

HUXLEY, A. New RHS. Dictionary of Gardening. London: Macmillan Press, 1992. 3000p.

IBRAHIM, S. M.M.; TAHA, L.S.; FARAHAT, M.M. Vegetative growth and chemical constituents of croton plants as affected by foliar application of benzyl adenine and gibberellic Acid. Journal of American Science, v.6, n. 7, p.126-130, 2010.

JACKSON, M. L. Soil Chemical Analysis. New Delhi: Prentice-Hall, 1973. 498p.

KOCHHAR, S.L.; SUKHBIR K.G. Plant Physiology: Theory and Applications. Cambridge: University Press, 2020. 800p.

LIMA, J.D; ANSANTE, N.F.; NOMURA, E.S.; FUZITANI, E.J.; DA SILVA, S.H. Growth and yield of anthurium in response to gibberellic acid. Ciência Rural, v.44, n.8, p.1327-1333, 2014. DOI: https://doi. org/10.1590/0103-8478cr20120586

MATAK, S.A.; KAVIANI, B.; HASHEMABADI, D. Changes in postharvest physio-biochemical characterustics and antioxidant enzymes activity of cut Alsteroemeria aurantiaca flower as affected by cycloheximide, coconut water and 6-benzyladenine. Biosciences Journal, v.33, n.2, p.321-332, 2017. DOI: https://doi.org/10.14393/BJv33n2-34381 
MAZHER, A.A.M.; ABDEL-AZIZ, N.G.; ELMAADAWY, E.I.; NASR, A.A.; EL-SAYED, S.M. Effect of gibberellic acid and paclobutrazol on growth and chemical composition of Schefflera arboricola plants. Middle-East Journal of Agriculture Research, v.3, n.4, p.782-792, 2014.

MOHAMED, Y.F.Y. Influence of different growing media and kristalon chemical fertilizer on growth and chemical composition of Areca Palm (Dypsis cabadae H. E. Moore) Plant. Middle East Journal of Applied, v.8, n.1, p.43-56, 2018.

MOUSA, G.T.; ABDUL-HAFEEZ, E.Y.; IBRAHIM, O.H.M. Response of gardenia plants grown under various growth media and ferrous sulfate application. Pakistan Journal of Agriculture Sciences, v.52, n.3, p.651-658, 2015 .

NETTO, A.T.; CAMPOSTRINI, E.; DEOLIVIERA, J.G.; BRESSAN-SMITH, R.E. 2005. Photosynthetic pigments, nitrogen, chlorophyll a fluorescence and SPAD-502 readings in coffee leaves. Scientia Horticulturae, v.104, n.2, p.199-209, 2005.

NGAPUI, R.; GOGOI, P.; CHOWLU, K.; VIJ, S.P. Effects of NPK and 6 benzylaminopurine on growth and flowering of two Orchid Genera. Journal of Plant Science Research, v.34, n.1, p.93-99, 2018. DOI: 10.32381/ JPSR.2018.34.01.10

ODENWALD, N.G.; TURNER, J.R. Identification, selection and use of southern plants for landscape design, 4ed. Baton Rouge: Claitor's Publishing Division, 2006. 193p.

PASCUAL, J.A.; CEGLIE, F.; TUZEL, Y.; KOLLER, M.; KOREN, A.; HITCHINGS, R.; TITTARELLI, F. Organic substrate for transplant production in organic nurseries. A review. Agronomy for Sustainable Development, v.38, n.35, p.1-23, 2018. DOI: https://doi.org/10.1007/s13593018-0508-4

PLACKETT, A.R.G.; WILSON, Z.A. Gibberellins and plant reproduction. In:Annual plant reviews online. p.323358, 2016, DOI: https://doi.org/10.1002/9781119312994. apr0540.
SARDOEI, A.S.; ZARINKOLAH, M.; MOHAMMADI, $\mathrm{H}$. Foliar applications of gibberellic acid and benzyladenine increase vines of 'White Butterfly' Syngonium podophyllum. International Journal of Farming and Allied Sciences, v.7, n.2, p.31-36, 2018.

SATISH, C.B.; MANJU, A.L. Plant Physiology, Development and Metabolism. New Delhi: Springer Nature, 2018. 1237p.

SHAHID, A.; HUSSAIN, R.; RIAZ A.; YOUNIS, A. Effect of different substrates on vegetative growth and quality of cast iron (Aspidistra elatior L.). International Journal of Biosciences, v. 10, n. 3, p. 297-308, 2017. DOI: http:// dx.doi.org/10.12692/ijb/10.3.297-308

STEEL, R.G.D.; TORRIE, J.H.; DICKEY, D.A. Principles and Procedures of Statistics. A Biometrical Approach, 3ed. New York: McGraw-Hill Inc., 1997. 666p.

TANDEL, M.H.; ANIMASAUN, D.A.; KRISHNAMURTHY, R. Growth and phytochemical composition of Adhatoda zeylanica in response to foliar application of growth hormones and urea. Journal of Soil Science and Plant Nutrition, v.18, n.3, p.881892, 2018. DOI: http://dx.doi.org/10.4067/S071895162018005002601

ULLAH, S.; ANWAR, S.; REHMAN, M.; KHAN, S.; ZAFAR, S.; LIU, L.; PENG, D. Interactive effect of gibberellic acid and NPK fertilizer combinations on ramie yield and bast fibre quality. Scientific Reports, v.7, n.1, p.1-9, 2017. DOI: https://doi.org/10.1038/s41598-01709584-5

YOUNIS, A.; RIAZ, A.; ZULFIQAR, F.; AKRAM, A.; KHAN, M.A.; TARIQ, U.; NADEEM, M.; AHSAN, M. Quality Lady Palm (Rhapis exclesaL.) production using various growing media. International Journal of Advances in Agriculture Sciences, v.1, n.1, p.1-9, 2016. 